Systematic Review

\title{
Is Vitamin D Supplementation Effective for Low Back Pain? A Systematic Review and Meta-Analysis
}

Joshua R. Zadro, BAppSC1, Debra Shirley, PhD1, Maneula Ferreira, PhD², Ana Paula Carvalho Silva, MSc${ }^{1}$, Sarah E. Lamb, PhD ${ }^{3}$, Cyrus Cooper, PhD², and Paulo $\mathrm{H}$. Ferreira, PhD ${ }^{1}$

\footnotetext{
From: ${ }^{1}$ Discipline of Physiotherapy, Faculty of Health Sciences, The University of Sydney, Sydney, Australia; ${ }^{2}$ Institute of Bone and Joint Research/The Kolling Institute \& School of Public Health, Sydney Medical School, The University of Sydney, Sydney, Australia; ${ }^{3}$ Nuffield Department of Orthopaedics, Rheumatology and Musclo-Skeletal Sciences, University of Oxford, Oxford

Address Correspondence: Joshua Robert Zadro, BApp Faculty of Health Sciences,

The University of Sydney 75 East Street Lidcombe, Sydney NSW 1825 , Australia E-mail: jzad3326@uni.sydney.edu.au

Disclaimer: There was no external funding in the preparation of this manuscript.

Conflict of interest: Each author certifies that he or she, or a member of his or her immediate family, has no commercial association

(i.e., consultancies, stock ownership, equity interest, patent/licensing arrangements, etc.) that might pose a conflict of interest in connection with the submitted manuscript.

Manuscript received: 06-02-2017

Revised manuscript received: 07-19-2017

Accepted for publication: 09-05-2017

Free full manuscript: www.painphysicianjournal.

com
}

Background: Low back pain (LBP) is the leading cause of years lived with disability worldwide. Current intervention strategies are failing to reduce the enormous global burden of LBP and are prompting researchers to investigate alternative management strategies, such as vitamin D supplementation. Vitamin D supplementation appears to down regulate pro-inflammatory cytokines which lead to pain and up regulate anti-inflammatory cytokines that reduce inflammation. These mechanisms might explain the increasing interest in the use of vitamin D supplementation for LBP.

Objectives: To determine whether vitamin D supplementation improves pain more than a control intervention for individuals with LBP.

Study Design: This study was conducted in accordance with the Preferred Reporting Items for Systematic Reviews and Meta-Analyses (PRISMA) statement.

Methods: We performed searches in numerous electronic databases combining key words relating to "vitamin D" and "LBP" until March 2017. Studies were included if they investigated vitamin D supplementation in participants with LBP, provided there was a comparison intervention. There was no restriction on the type of LBP, the intervention parameters investigated, or the type of clinical trial (e.g., randomized, non-randomized). Two reviewers independently performed the selection of studies, extracted data, rated the methodological quality of the included studies, and evaluated the overall quality of the evidence using the Grading of Recommendations Assessment, Delevopment, and Evaulation (GRADE) approach.

Results: After screening 3,534 articles, 8 clinical trials were included in this systematic review. There is very low quality evidence (based on the GRADE approach) that vitamin D supplementation is not more effective than any intervention (including placebo, no intervention, and other conservative/ pharmacological interventions) (continuous pain measures [0-100]: mean difference [MD] $=-2.65$ 95\% confidence interval $[\mathrm{Cl}]$ : -10.42 to $5.12, P=0.504, n=5$; self-reported reduction in pain pooled odds ratio $[\mathrm{OR}]=1.07,95 \% \mathrm{Cl}: 0.35$ to $3.26, P=0.906, \mathrm{n}=5$ ) or placebo/no intervention for individuals with LBP (continuous pain measures: $M D=1.29,95 \% \mathrm{Cl}:-3.81$ to $6.39, P=0.620$, $\mathrm{n}=4$; self-reported reduction in pain: pooled $\mathrm{OR}=1.53,95 \% \mathrm{Cl}: 0.38$ to $6.20, P=0.550, \mathrm{n}=4$ ) where ' $n$ ' is the number of studies included in the meta-analysis. These results did not change when we stratified the meta-analyses by the type of vitamin supplementation (vitamin D3 vs. alfacalcidol) or the type of LBP (non-specific vs. LBP resulting from osteoporosis or vertebral fractures).

Limitations: The overall quality of evidence was "very low" due to the poor methodological quality and small sample sizes of the included studies.

Conclusions: Vitamin D supplementation is not more effective than placebo, no intervention, or other conservative/pharmacological interventions for LBP (based on very low quality evidence). These results are consistent, regardless of the type of LBP or vitamin D supplementation. Until well-designed and adequately powered clinical trials suggest otherwise, the prescription of vitamin D for LBP cannot be recommended.

PROSPERO Registration No: CRD42016046874. www.crd.york.ac.uk/PROSPERO/display_record asp?ID = CRD42016046874

Key words: Vitamin D, low back pain, chronic low back pain, alfacalcidol, osteoporosis, vertebral fractures, serum 25-hydroxyvitamin D, systematic review

Pain Physician 2018; 21:121-145 
ow back pain (LBP) is the leading cause of years lived with disability worldwide (1), with the total yearly costs of LBP estimated at $\$ 9$ billion in Australia (2) and $€ 300$ billion for the whole of Europe (3). The majority of LBP cases presenting to primary care are classified as 'non-specific' ( 85\%) (4), as there is a poor correlation between symptoms and structural abnormalities identified by medical imaging $(5,6)$. A small percentage of individuals may present with LBP that can be attributed to a structural pathology $(<5 \%)(7,8)$, such as osteoporosis or vertebral fractures, and can experience significant pain and disability (9). Numerous intervention strategies have been investigated for non-specific LBP and are recommended in most evidence-based clinical practice guidelines (10), such as structured exercise programs (11) and advice to remain active (12). In addition, numerous conservative $(13,14)$, pharmacological $(15,16)$, and surgical interventions (17) have been investigated for the management of LBP resulting from osteoporosis or vertebral fractures. However, despite an abundance of research investigating different types and doses of these interventions (18-22), the analgesic effects are modest at best (23), and are failing to reduce the enormous global burden of LBP (1). With this in mind, it may be time to consider alternative interventions, rather than investigating procedural adjustments of those already established. One of the current and popular alternative treatments for painful conditions (24), including LBP $(25,26)$, is vitamin D supplementation.

Vitamin $D$ is an essential hormone for optimal bone, neuromuscular, and immune function. Skin exposure to sunlight is the main pathway by which vitamin D is synthesized, although supplementation and some foods may provide an additional source (27). Vitamin $D$ is commonly recommended by medical professionals for individuals with osteoporosis (28), as it can increase bone mineral density through calcium absorption and bone mineralization (29). Nevertheless, there is conflicting evidence regarding the effect vitamin $D$ has on pain. For participants with LBP resulting from osteoporosis or vertebral fractures, increases in bone mineral density may provide analgesic effects (29). However, other proposed mechanisms by which vitamin $D$ supplementation could reduce pain include: a down-regulation of proinflammatory cytokines which lead to pain or an upregulation of anti-inflammatory cytokines that reduce inflammation (30). Two recent systematic reviews investigated the effectiveness of vitamin D supplementation compared to placebo for chronic painful conditions (e.g., knee osteoarthritis, rheumatoid arthritis, fibromyalgia, and LBP) $(31,32)$. One review found no consistent effect of vitamin $D$ compared to placebo but did not perform a meta-analysis due to substantial heterogeneity between studies in regards to the methodological quality, chronic painful condition investigated, and vitamin D dosage (32). In contrast, another systematic review performed a meta-analysis despite between-study heterogeneity and found that vitamin D supplementation was slightly more effective than placebo when considering changes in pain from baseline (mean difference $[M D]=-0.57,95 \%$ confidence interval $[\mathrm{Cl}]:-1.00$ to $-0.15, P=0.007)$. However, vitamin $D$ supplementation was not more effective than placebo when considering post-intervention pain scores (MD $=-0.06,95 \% \mathrm{Cl}:-0.44$ to $0.33, P=0.780$ ) or self-reported reductions in pain (relative risk $=1.38,95 \% \mathrm{Cl}: 0.93$ to $2.05, \mathrm{P}=0.110$ ). $\mathrm{A}$ significant limitation of these reviews is a general focus on chronic painful conditions, as this can introduce significant heterogeneity between study findings and neglect different disease presentations (e.g., acute vs. chronic). This highlights the need to investigate the effects of vitamin D supplementation in specific conditions, such as LBP. Vitamin D supplementation is easily accessible, cheap, and has minimal side effects, which may explain why its use for the management of LBP is gaining increasing attention $(25,26)$. Therefore, to better understand whether vitamin $D$ supplementation is effective for LBP, it is important to consider all presentations of the condition (e.g., non-specific LBP, LBP resulting from osteoporosis or vertebral fractures). The aim of this systematic review is to investigate the effectiveness of vitamin D supplementation for LBP.

\section{Methods}

\section{Search Strategy}

This systematic review was conducted in accordance with the Preferred Reporting Items for Systematic Reviews and Meta-Analyses (PRISMA) statement (33), and the protocol was registered on PROSPERO (Registration No: CRD42016046874). MEDLINE, CINAHL, EMBASE, AMED, WEB OF SCIENCE, and SCOPUS databases were searched to identify eligible studies from the earliest record to March 2017. Our search combined key words related to vitamin D (e.g., "alfacalcidol" OR "ergocalciferol" OR "1-alpha hydroxyvitamin D3," etc.) and LBP (e.g., "back ache" OR "back pain" OR "spinal pain," etc.) and remained sensitive to the study design to capture all types of clinical trials (e.g., randomized 
controlled trials, non-randomized trials) (Appendix 1). Citation tracking was performed for all studies found by electronic searches, and the reference lists of the included studies were hand-searched to identify studies missed by the above processes.

\section{Study Selection}

Two reviewers (JZ and AS) independently screened the titles, abstracts, and selected full text articles for inclusion using a study eligibility form based on items from the inclusion/exclusion criteria. Disagreements were resolved by discussion and consultation with a third reviewer (DS). There was no restriction on the language or geographic setting of the study, although studies not published in English were excluded when an appropriate translation was not available. There was no restriction on the age or gender of participants or the type of publication (e.g., conference abstract or dissertation).

Studies were included if they assessed any measure of pain (e.g., visual analog scale [VAS], Face Scale, numeric rating scale [NRS]), function (e.g., Roland Morris Disability Questionnaire, Patient-Specific Functional Scale), or subjective measure of improvement in people with LBP following vitamin D supplementation. We included studies investigating participants with non-specific LBP and LBP resulting from osteoporosis (including studies which enrolled participants with vertebral compression fractures) but excluded studies investigating participants with evidence of nerve root compression or a diagnosis of serious spinal pathology, such as metastatic disease or cauda equina syndrome. There was no restriction on the intervention parameters (e.g., type of supplement, dosage, duration, and administration route). Studies with no comparison intervention or where vitamin $D$ was used in conjunction with another active therapy (e.g., calcium supplementation, nonsteriodal anti-inflammatory drugs) were excluded, unless the additional active therapy was identical for both the intervention and control group (e.g., vitamin $\mathrm{D}$ and calcium supplementation vs. calcium supplementation alone). We included both randomized and non-randomized trials to get a broader overview of the efficacy of vitamin D supplementation for LBP. We excluded single-arm trials (no comparison), case series, and case reports.

\section{Methodological Quality}

Two reviewers (JZ and AS) independently assessed the methodological quality of the included studies and the overall quality of evidence and strength of recommendation, resolving any disagreement by consensus. For studies satisfying the eligibility criteria, we used the valid and reliable PEDro scale to score the methodological quality of each study (34). The Grading of Recommendations Assessment, Development, and Evaluation (GRADE) approach was used to evaluate the overall quality of evidence and the strength of recommendation (35). The quality of evidence was downgraded for each of the following 5 factors encountered: limitations in the design and implementation [ $>25 \%$ of the trial, weighted by their sample size, scored $<7$ on the PEDro scale (36)], indirectness of evidence (trial design and objective not concordant), unexplained heterogeneity $\left(I^{2}>50 \%\right)$, imprecision of results [comparisons with less than 400 participants were deemed "low quality evidence" $(36,37)]$, and high probability of publication bias [assessed using Egger's funnel plot (38)]. We did not assess publication bias when a meta-analysis was not possible or if less than 10 studies were included in the meta-analysis $(38,39)$. The quality of evidence was downgraded by one level if both reviewers (JZ and AS) judged the limitation was 'serious' or by 2 levels if it was judged as 'very serious.' The following was used to define the quality of evidence for each outcome (40):

- High quality: very confident the true effect lies close to the effect estimate with no known or suspected reporting biases; all domains were fulfilled

- Moderate quality: moderately confident the true effect lies close to the effect estimate with some possibility that it is substantially different; one domain was not fulfilled

- Low quality: limited confidence the true effect lies close to the effect estimate; 2 domains were not fulfilled

- Very low quality: very little confidence the true effect lies close to the effect estimate; 3 domains were not fulfilled.

\section{Data Extraction}

Data was independently extracted from the included studies by 2 reviewers (JZ and AS) using a standardized data extraction form to collect relevant information on participant characteristics (age and gender), study setting (e.g., hospital or community), sample size, features specific to the study design, type and duration of LBP, baseline and follow-up measures of pain and function (all time-points), intervention parameters (type of supplementation, dosage, duration, and ad- 
ministration route), the comparison intervention, loss to follow-up, and the incidence of adverse events.

\section{Statistical Analysis}

We extracted data on the between-group MD (continuous data) or odds ratio (OR) (dichotomous data) and $95 \% \mathrm{Cl}$ at all time-points (including change scores when reported) for measures of pain and function following a course of vitamin D supplementation in individuals with LBP. Scores on pain and function outcomes were transformed to a 0-100 scale, where 0 represents no pain/poor function and 100 represents highest pain/ highest level of function. We attempted to calculate a pooled weighted MD or OR $(95 \% \mathrm{Cl})$ when studies were considered sufficiently homogenous using Comprehensive Meta-Analysis Version 3.0 (Biostat, Englewood, NJ). For continuous data, when pre- and post-intervention means and standard deviation (SD) were available, but the $\mathrm{MD}$ and $95 \% \mathrm{Cl}$ were not reported, these were calculated in the meta-analysis software. For dichotomous outcomes, when pre- and post-intervention event data (percentages) were available, but the OR and $95 \% \mathrm{Cl}$ were not reported, these were calculated in the metaanalysis software. For outcomes measured on a 0-100 scale (e.g., VAS), a 20-point between-group difference was considered clinically meaningful (41).

Heterogeneity was assessed using the $\mathrm{I}^{2}$ statistic and was considered low where $\mathrm{I}^{2}<25 \%$, moderate where $I^{2} \geq 50 \%$, and high where $I^{2} \geq 75 \%$ (42). Fixed effects models were used when $\mathrm{I}^{2}<50 \%$, and random effects models were used when $I^{2} \geq 50 \%$. If there were enough studies investigating various types of LBP (e.g., chronic LBP, acute LBP, LBP resulting from osteoporosis or vertebral fractures, etc.), doses of vitamin $D$, types of supplementation (e.g., alfacalcidol/calcitriol vs. vitamin D3), or where there were between study differences in participant demographics (e.g., age, gender, etc.) and the comparison intervention (e.g., placebo, no intervention, conservative/pharmacological interventions), we stratified meta-analyses accordingly (sensitivity analysis).

\section{Results}

\section{Description of Studies}

We identified 3,534 articles through our database searches with 2 articles identified through hand-searching the reference lists of included studies $(43,44)$ (Fig. 1.). Following the removal of duplicates, 2 reviewers (JZ and AS) independently screened the articles' titles and abstracts and screened the full-text of 102 articles. A total of 8 clinical trials were eligible for inclusion in this review, with available data from 747 participants. The characteristics of the included studies can be found in Table 1. We included 4 published randomized controlled trials $(25,44-46), 2$ conference abstracts of randomized controlled trials $(47,48)$, one randomized cross-over trial (49), and one non-randomized controlled trial (50). The PEDro scale was used to score the methodological quality of each study and ranged from 4 to 8 (Table 2). The 2 conference abstracts were not scored as per the criteria for including clinical trials in PEDro (51). The most common methodological limitations were lack of therapist $(n=5)$ and assessor $(n=3)$ blinding, no intention-to-treat analysis ( $n=6)$, and no attempt to conceal group allocation $(n=4)$.

The assessment of LBP differed between studies with 4 studies including participants who reported the presence of any $\operatorname{LBP}(44-47,50), 2$ studies including participants reporting chronic LBP (lasting for at least 3 months) $(25,49)$, and one study including participants reporting LBP for at least 2 weeks (48). In addition, 5 of the studies only investigated participants with LBP resulting from osteoporosis or vertebral fractures $(44,45,47,48,50)$, while 3 studies $(25,46,49)$ only investigated participants with non-specific LBP (2 studies investigated chronic non-specific $\operatorname{LBP}(25,49)$ and one study did not specify the duration of LBP symptoms at baseline (46)). There were differences between the settings for each study, with 4 studies recruiting participants from hospitals (2 in Japan $(45,50)$, one in Iran (25), and one in Greece (44)), one study recruiting participants from 10 general practices in the Netherlands (49), one study recruiting participants from a local immigrant activity centre in Norway (46), and 2 studies (conference abstracts) not reporting the source of participants $(47,48)$. The intervention parameters differed across studies with 3 studies $(25,46,49)$ prescribing vitamin D3 for individuals with non-specific LBP (dosage ranging from 25-179 ug daily and duration ranging from $6-16$ weeks) and 5 studies $(44,45,47,48,50)$ prescribing alfacalcidol (or calcitriol in one study (47)) for individuals with LBP resulting from osteoporosis or vertebral fractures (dosage ranging from 1-1000 ug daily and duration ranging from 1-24 months). Co-interventions included the prescription of oral cyclical etidronate or oral celecoxib and advice to take calcium supplementation, perform home exercises, or seek physiotherapy or additional analgesics if required. Comparison interventions varied according to the presentation of LBP. All 
Is Vitamin D Supplementation Effective for Low Back Pain?

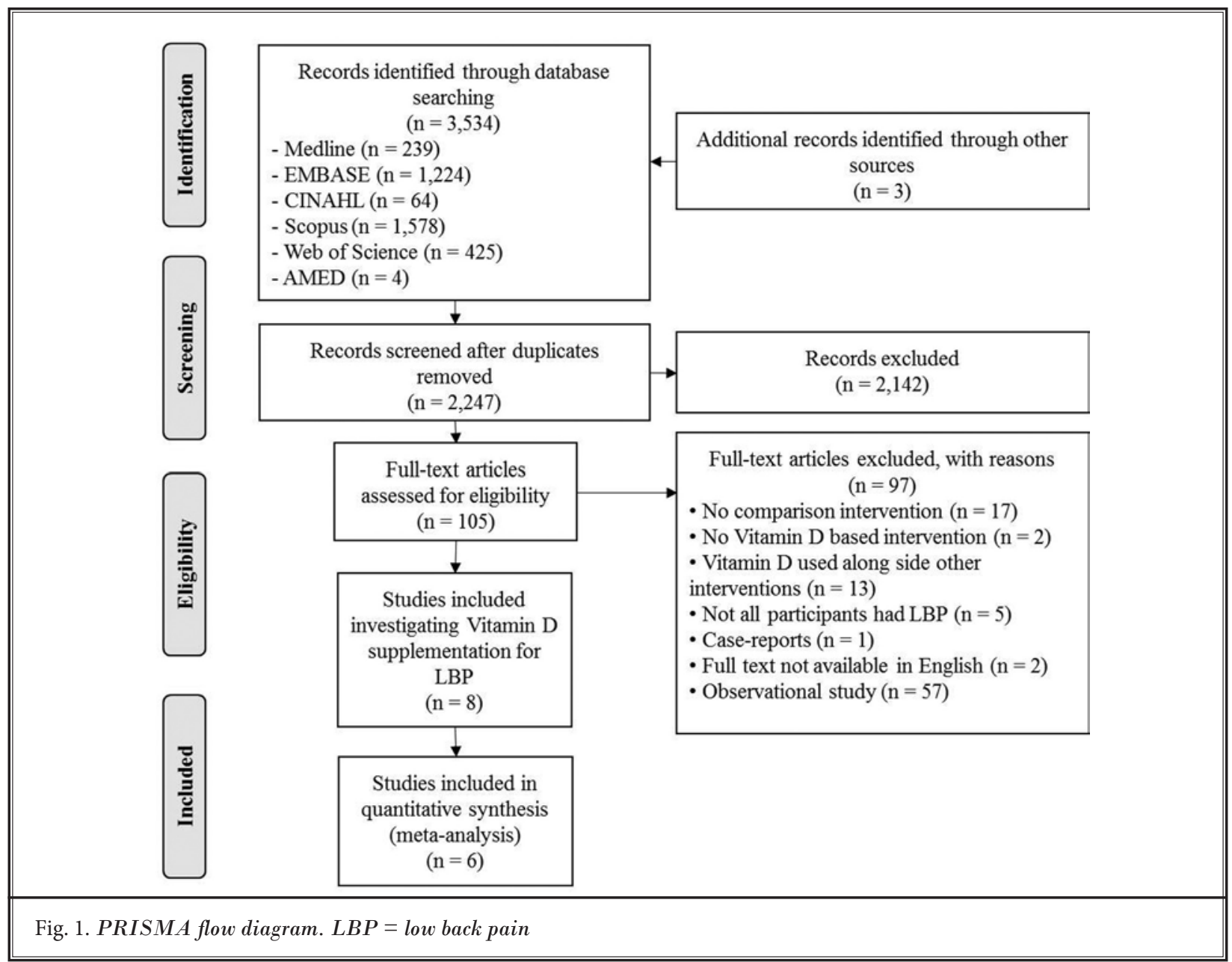

studies investigating participants with non-specific LBP used a placebo as the comparison intervention (with the same packaging, appearance, and taste). For studies investigating participants with LBP resulting from osteoporosis or vertebral fractures, the comparison interventions included: placebo, no intervention, and other conservative/pharmacological interventions (oral risedronate, oral raloxifene, intramuscular injections of eel calcitonin, or intramuscular injections of nandrolone decanoate). All of the included studies assessed pain, with some using multiple outcome measures (e.g., VAS and percentage of participants reporting clinical improvements). Five studies assessed pain using the VAS $(0-100)(25,46,48-50)$, one study using the Face Scale (45), one study using a 5 -point scale (where ' 0 ' $=$ no pain and ' 5 ' = very severe pain) (44), 4 studies using the percentage of participants that improved from baseline $(25,44,47,49)$, and one study reporting the proportion of participants still in pain at follow-up (46). Function was not assessed in any study. One conference abstract (48) failed to report sufficient data to be included in the meta-analysis, while the results from the other conference abstract (47) were only included in the metaanalyses of dichotomous outcomes since their outcome was the presence of a reduction in pain from baseline. We did not include the non-randomized controlled trial in any meta-analysis (50).

\section{Overall Effectiveness of Vitamin D Supplementation}

The overall pooling showed that vitamin D supplementation had no effect on pain levels for LBP when compared to any intervention (including placebo, no intervention, and other conservative/pharmacological interventions) ( $\mathrm{MD}=-2.65,95 \% \mathrm{Cl}:-10.42$ to $5.12, P=$ $0.504, n=5$ ) (Fig. 2) or when only compared to pla- 
Pain Physician: March/April 2018; 21:121-145

\begin{tabular}{|c|c|c|c|c|c|c|}
\hline 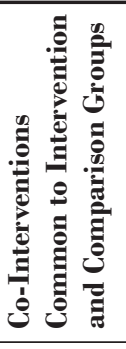 & 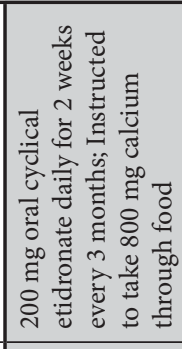 & \begin{tabular}{|l} 
\\
$\overleftrightarrow{Z}$ \\
\end{tabular} & 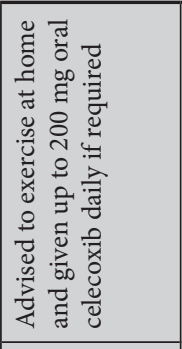 & $\overleftrightarrow{\mathbb{Z}}$ & 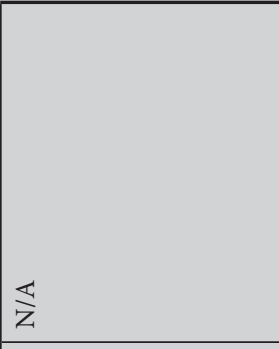 & 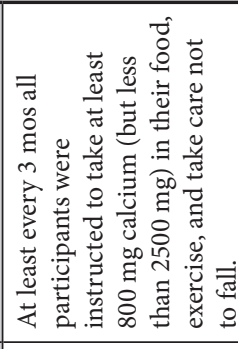 \\
\hline 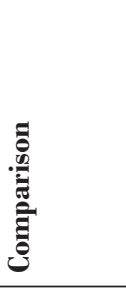 & 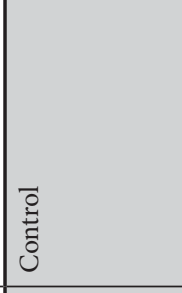 & $\begin{array}{l}8 \\
\stackrel{\Xi}{\tilde{U}} \\
\frac{\pi}{2}\end{array}$ & 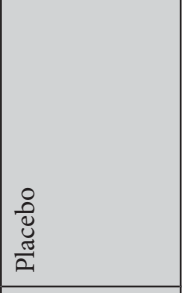 & $\begin{array}{l}0 \\
\frac{8}{\tilde{U}} \\
\frac{\tilde{\pi}}{2}\end{array}$ & 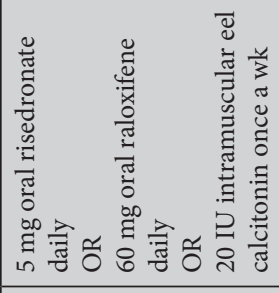 & 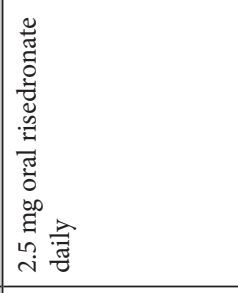 \\
\hline 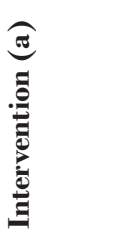 & 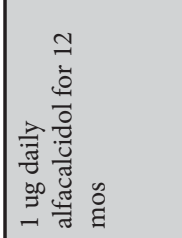 & 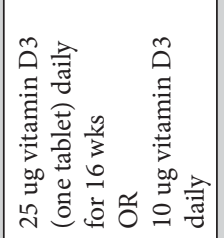 & 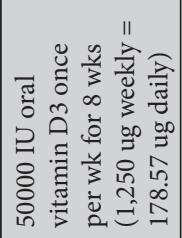 & 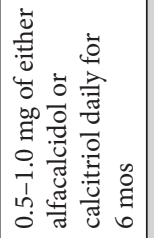 & 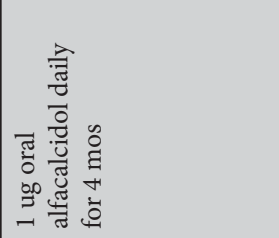 & 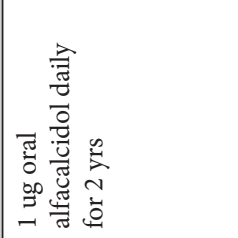 \\
\hline 言 & 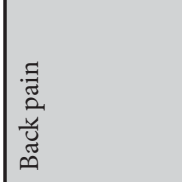 & 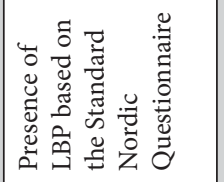 & 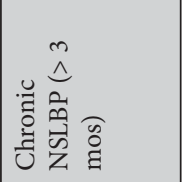 & 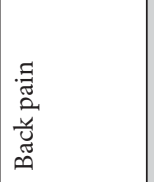 & 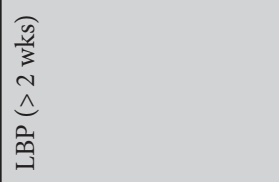 & 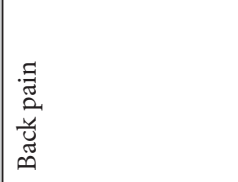 \\
\hline$\Rightarrow$ & 아 & $\stackrel{\vec{n}}{\sim}$ & in & $\stackrel{\sim}{\sim}$ & g & $\stackrel{8}{\circ}$ \\
\hline 泀 & 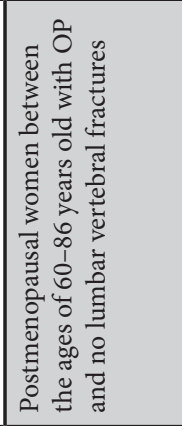 & 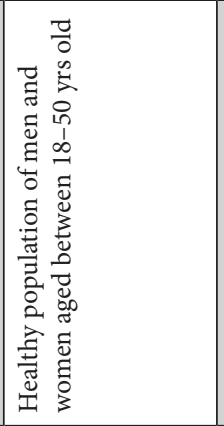 & 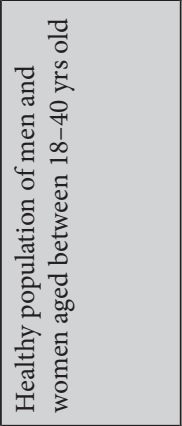 & 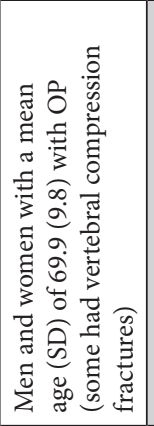 & 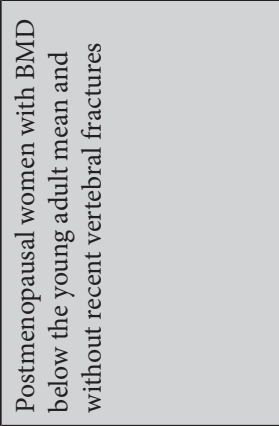 & 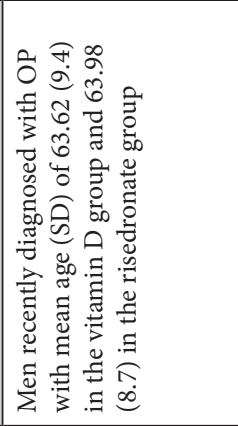 \\
\hline 尝 & 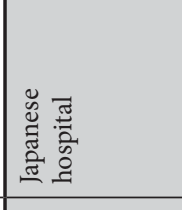 & 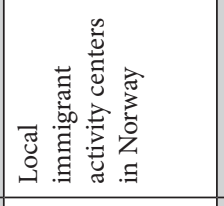 & 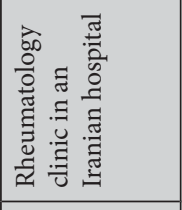 & 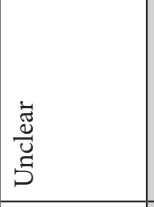 & $\begin{array}{l}\overrightarrow{\tilde{J}} \\
\overline{\breve{J}} \\
\tilde{5}\end{array}$ & 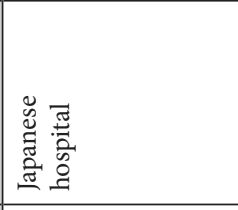 \\
\hline 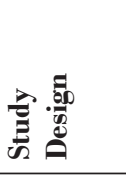 & $\tilde{\mathscr{q}}$ & $\underset{\widetilde{\Psi}}{\dot{y}}$ & $\underset{\widetilde{q}}{\breve{q}}$ & 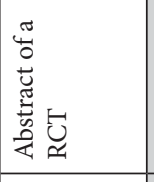 & 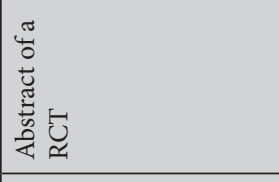 & 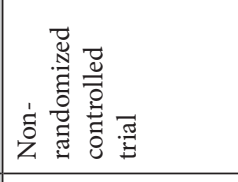 \\
\hline 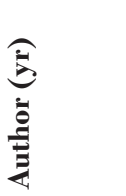 & 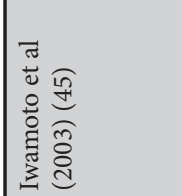 & 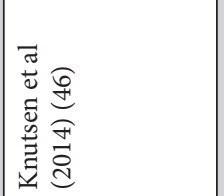 & 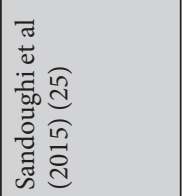 & 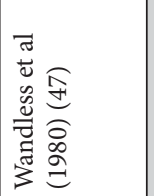 & 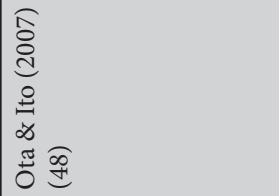 & 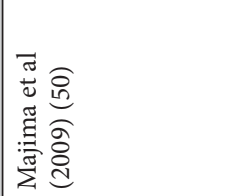 \\
\hline
\end{tabular}




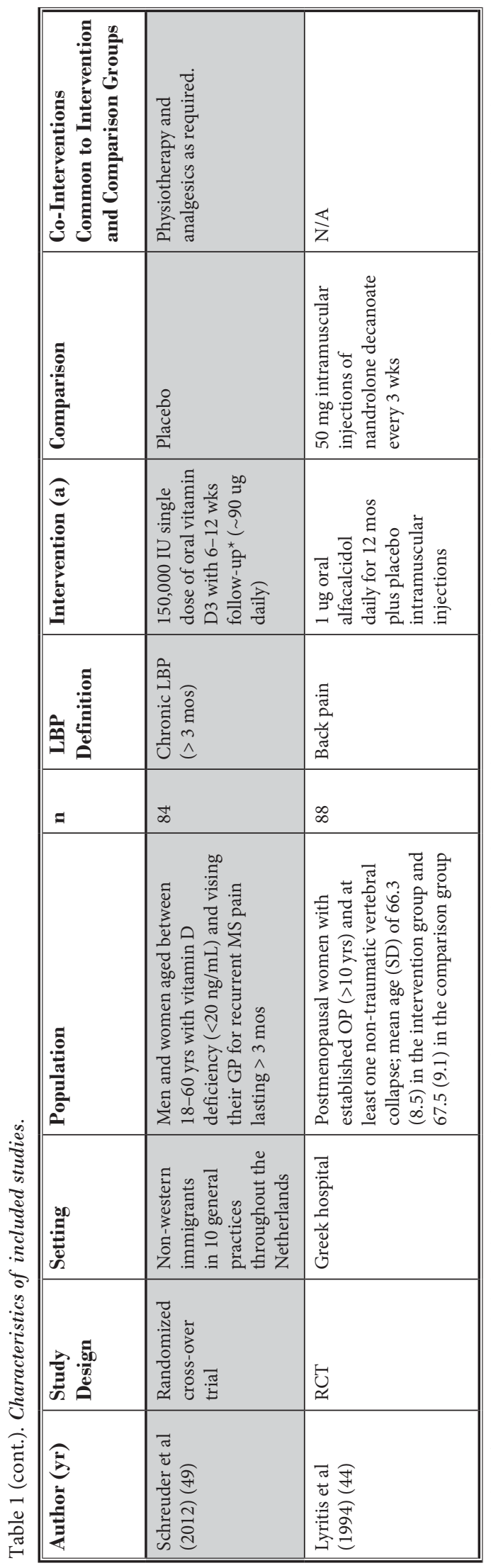

cebo/no intervention (MD $=1.29,95 \% \mathrm{Cl}:-3.81$ to 6.39, $P=0.620, n=4$ ) (Fig. 3), where ' $n$ ' is the total number of studies and a positive MD favors the intervention group. When improvement was based on a self-reported reduction in pain from baseline, there was no difference between vitamin D supplementation and any intervention (including placebo and other conservative/pharmacological interventions) (pooled OR $=1.07,95 \% \mathrm{Cl}: 0.35$ to $3.26, P=0.906, \mathrm{n}$ =5) (Fig. 4) or placebo only (pooled OR = 1.53, 95\% $\mathrm{Cl}$ : 0.38 to $6.20, P=0.550, \mathrm{n}=4$ ) (Fig. 5), where an OR > 1 indicates a higher likelihood of improvement for individuals receiving vitamin $D$ supplementation. The follow-up time-points varied between studies included in the meta-analyses, ranging from 6 weeks to 24 months (Table 1). The quality of the evidence for all meta-analyses was downgraded to "very low" due to imprecision of the results (all comparisons had a sample size less than 400) and limitations in study design and implementation ( $>25 \%$ of the studies, weighted by their sample size, scored $<7$ on the PEDro scale). As outlined previously, the types of LBP and vitamin D supplementation varied across studies, and we performed a number of sensitivity analyses accordingly. Across all included studies, individuals with nonspecific LBP were prescribed vitamin D3, while individuals with LBP resulting from osteoporosis/vertebral fractures were prescribed alfacalcidol (or calcitriol in one study (47)).

\section{Vitamin D for Non-Specific LBP}

In studies investigating individuals with non-specific LBP, vitamin D supplementation had no effect on pain (compared to placebo), regardless of symptom duration, when pain was measured on a continuous scale (all non-specific LBP: MD $=1.90,95 \% \mathrm{Cl}:-7.06$ to $10.86, P=0.678, \mathrm{n}=3$; chronic non-specific LBP only: $\mathrm{MD}=0.59,95 \% \mathrm{Cl}:-12.67$ to $13.84, P=0.931$, $\mathrm{n}=2$ ) (Fig. 6), or when improvement was based on a self-reported reduction in pain from baseline (all non-specific LBP: pooled OR $=0.85,95 \% \mathrm{Cl}$ : 0.28 to 2.60, $P=0.775, \mathrm{n}=3$; chronic non-specific LBP only: pooled OR $=1.02,95 \% \mathrm{Cl}: 0.14$ to $7.76, P=0.982, \mathrm{n}=$ 2) (Fig. 7).

\section{Vitamin D for LBP Resulting from Osteoporosis or Vertebral Fractures}

Similarly, vitamin D (alfacalcidol/calcitriol) had no effect on pain in studies investigating individuals with LBP resulting from osteoporosis or vertebral fractures when compared to any intervention (including no intervention and other conservative/pharmacological 
Table 2. Methodological quality of the included studies*.

\begin{tabular}{|c|c|c|c|c|c|c|c|c|c|c|c|c|c|}
\hline \multirow{2}{*}{ Author (yr) } & \multicolumn{11}{|c|}{ PEDro Scale Checklist } & \multirow{2}{*}{$\begin{array}{l}\text { Total } \\
\text { Score }\end{array}$} & \multirow{2}{*}{$\mathbf{n}$} \\
\hline & 1 & 2 & 3 & 4 & 5 & 6 & 7 & 8 & 9 & 10 & 11 & & \\
\hline $\begin{array}{l}\text { Iwamoto et al } \\
(2003)(45)\end{array}$ & + & + & - & + & - & - & - & - & - & + & + & 4 & 40 \\
\hline $\begin{array}{l}\text { Knutsen et al } \\
(2014)(46)\end{array}$ & + & + & + & + & + & - & + & + & - & + & + & 8 & 76 \\
\hline $\begin{array}{l}\text { Sandoughi et al } \\
(2015)(25)\end{array}$ & + & + & + & - & + & - & + & + & - & - & + & 6 & 53 \\
\hline $\begin{array}{l}\text { Majima et al } \\
(2009)(50)\end{array}$ & + & - & - & + & - & - & - & + & - & + & + & 4 & 62 \\
\hline $\begin{array}{l}\text { Schreuder et al } \\
(2012)(49)\end{array}$ & + & + & - & - & + & + & - & $+^{* *}$ & - & - & + & 5 & 50 \\
\hline $\begin{array}{l}\text { Lyritis et al (1994) } \\
\text { (44) }\end{array}$ & - & + & - & + & + & - & + & - & - & - & + & 5 & 69 \\
\hline $\begin{array}{l}\% \text { of Studies } \\
\text { Fulfilling Each } \\
\text { Item }\end{array}$ & $83.3 \%$ & $83.3 \%$ & $33.3 \%$ & $66.7 \%$ & $66.7 \%$ & $16.7 \%$ & $50 \%$ & $66.7 \%$ & $0 \%$ & $50.0 \%$ & $100 \%$ & & \\
\hline
\end{tabular}

$\mathrm{n}=$ number of participants who entered the meta-analyses.

${ }^{*}$ Wandless et al (47) (1980) and Ota \& Ito (48) (2007) were conference abstracts and were not included in this table due to insufficient data.

${ }^{*}$ Data from the first 6 weeks (before the cross-over) where the drop-out was $3.8 \%$ for individuals with LBP.

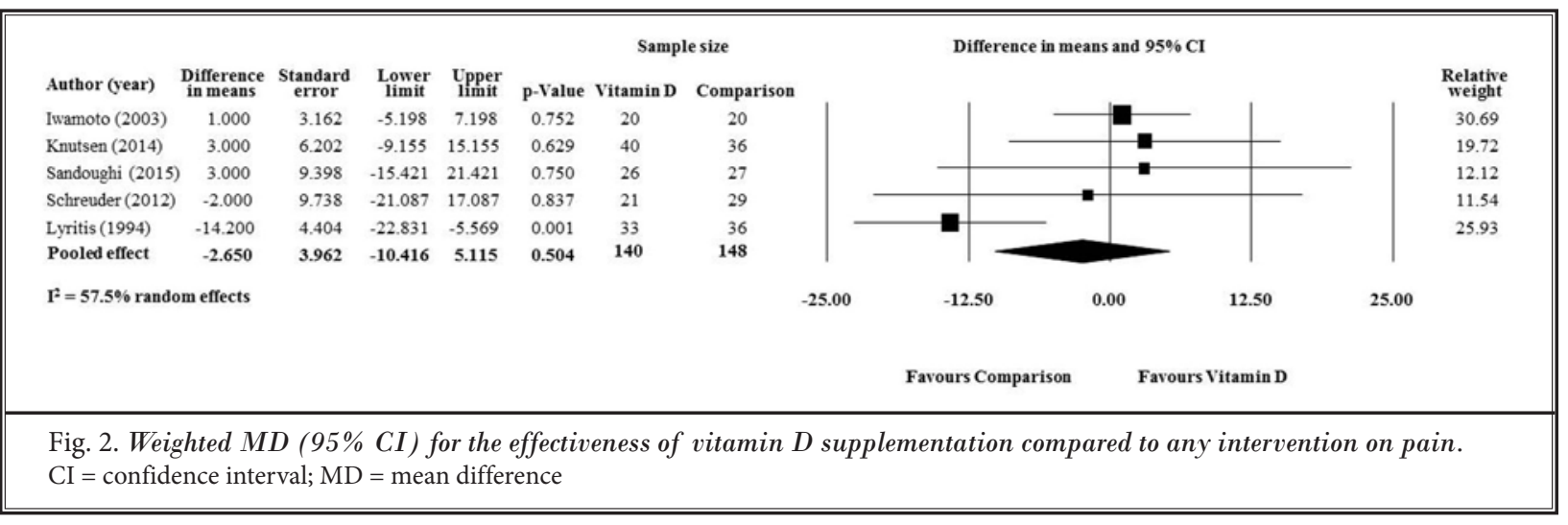

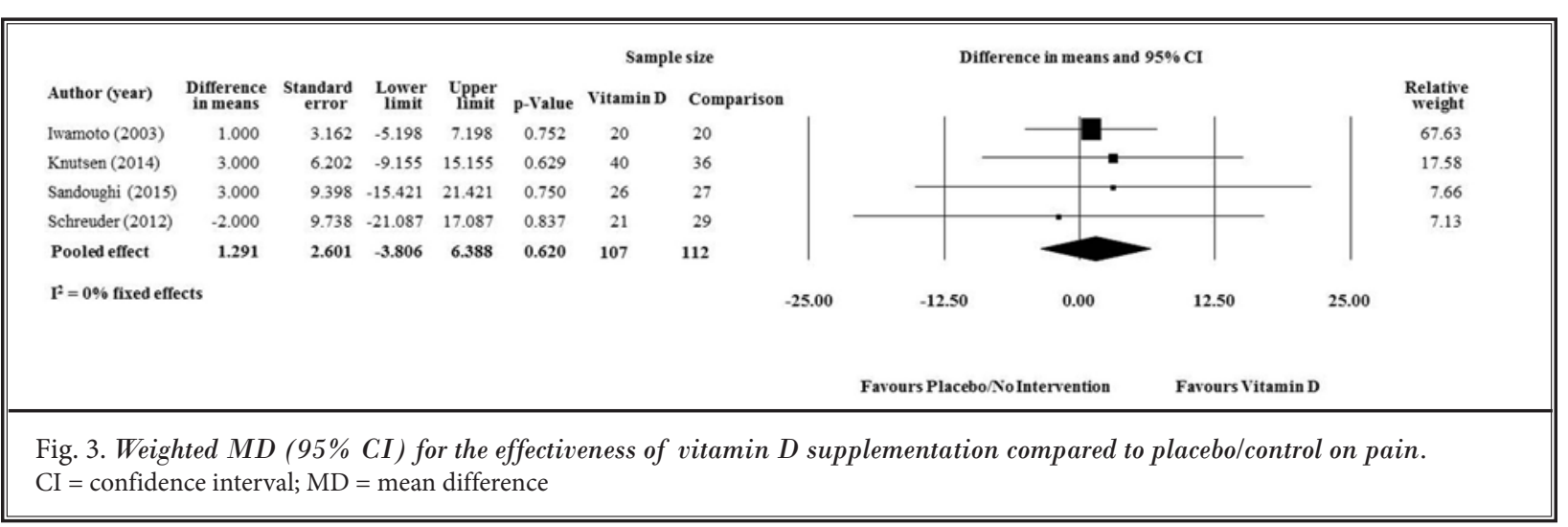


Is Vitamin D Supplementation Effective for Low Back Pain?

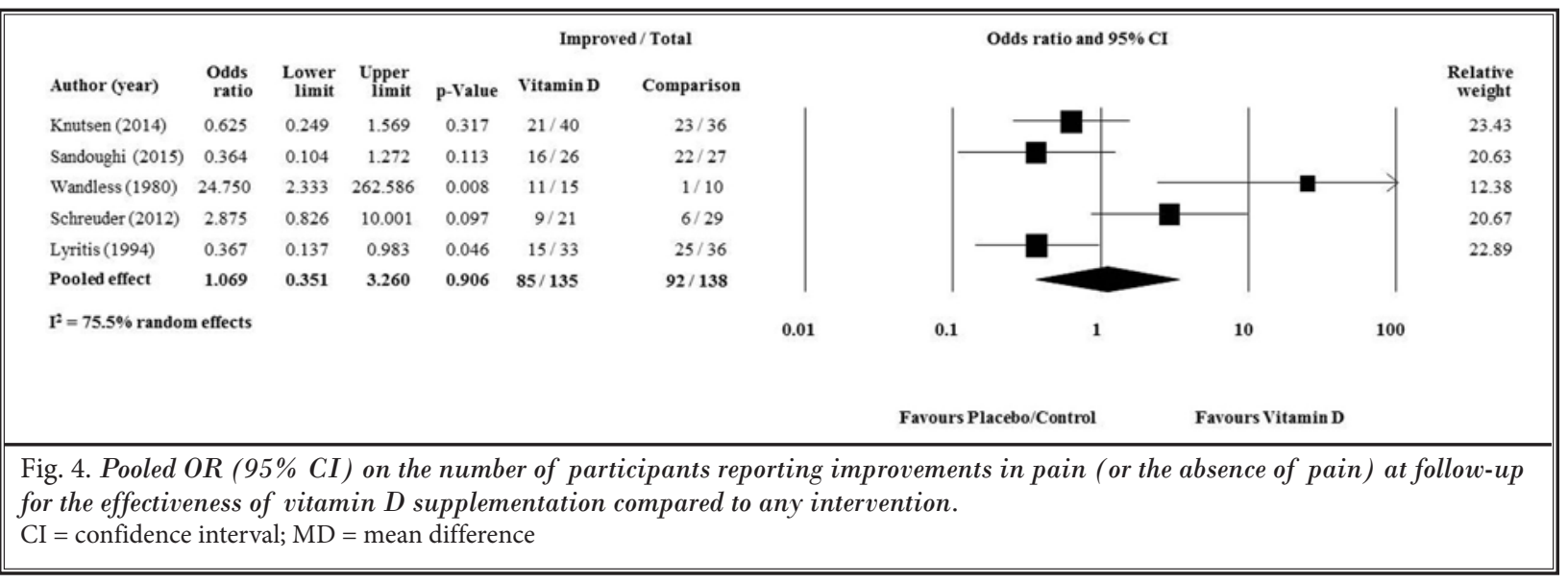

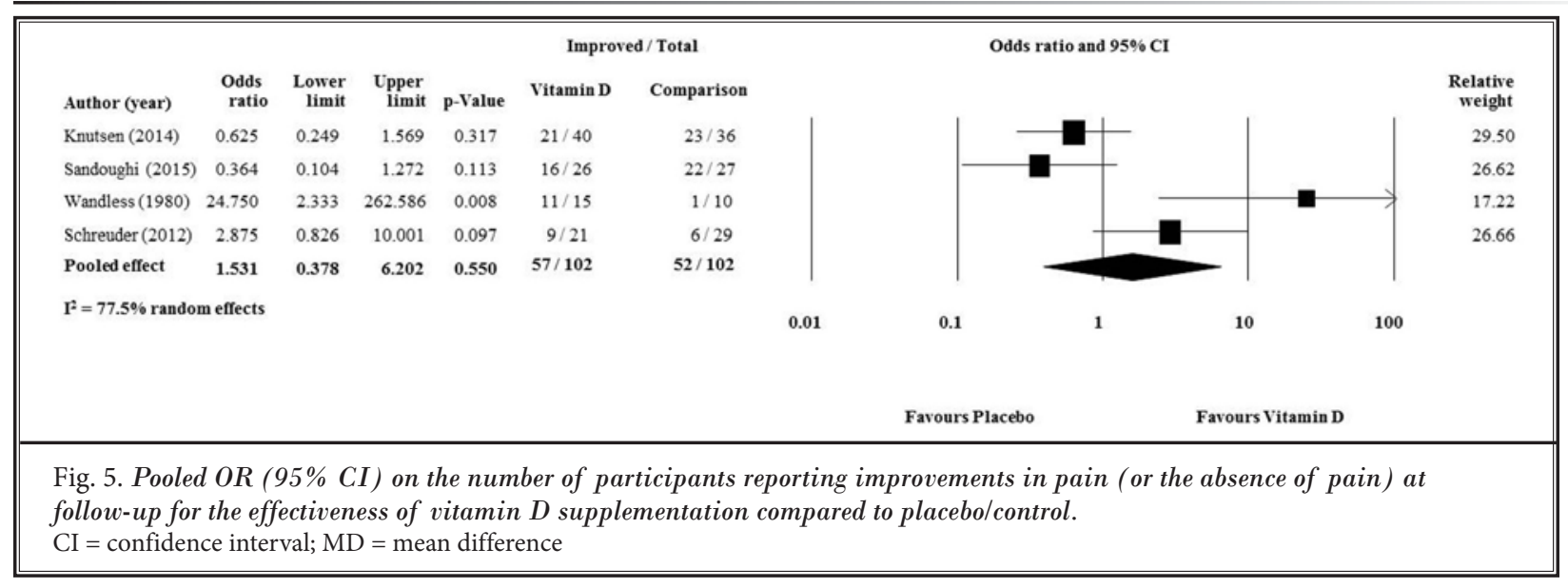

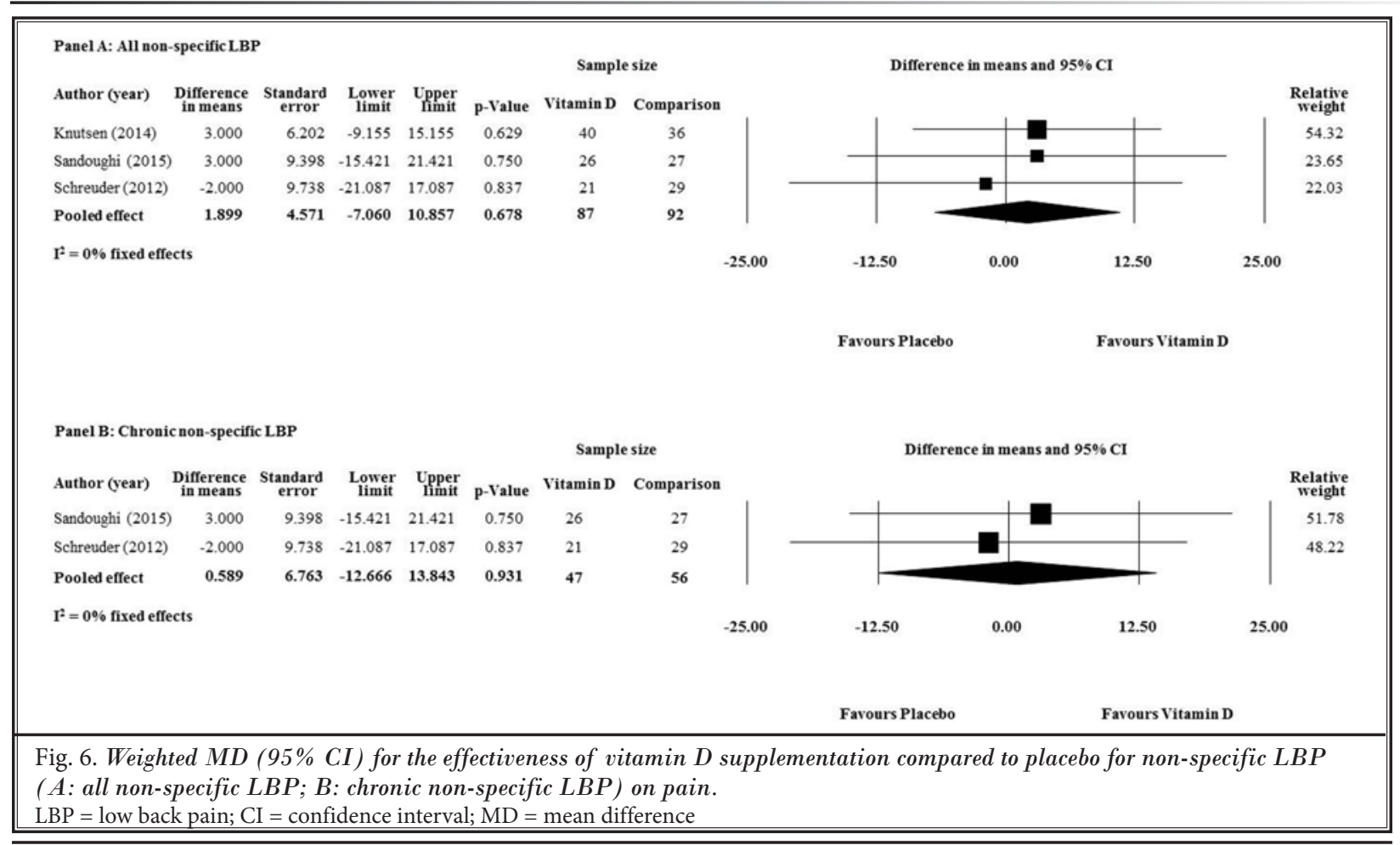




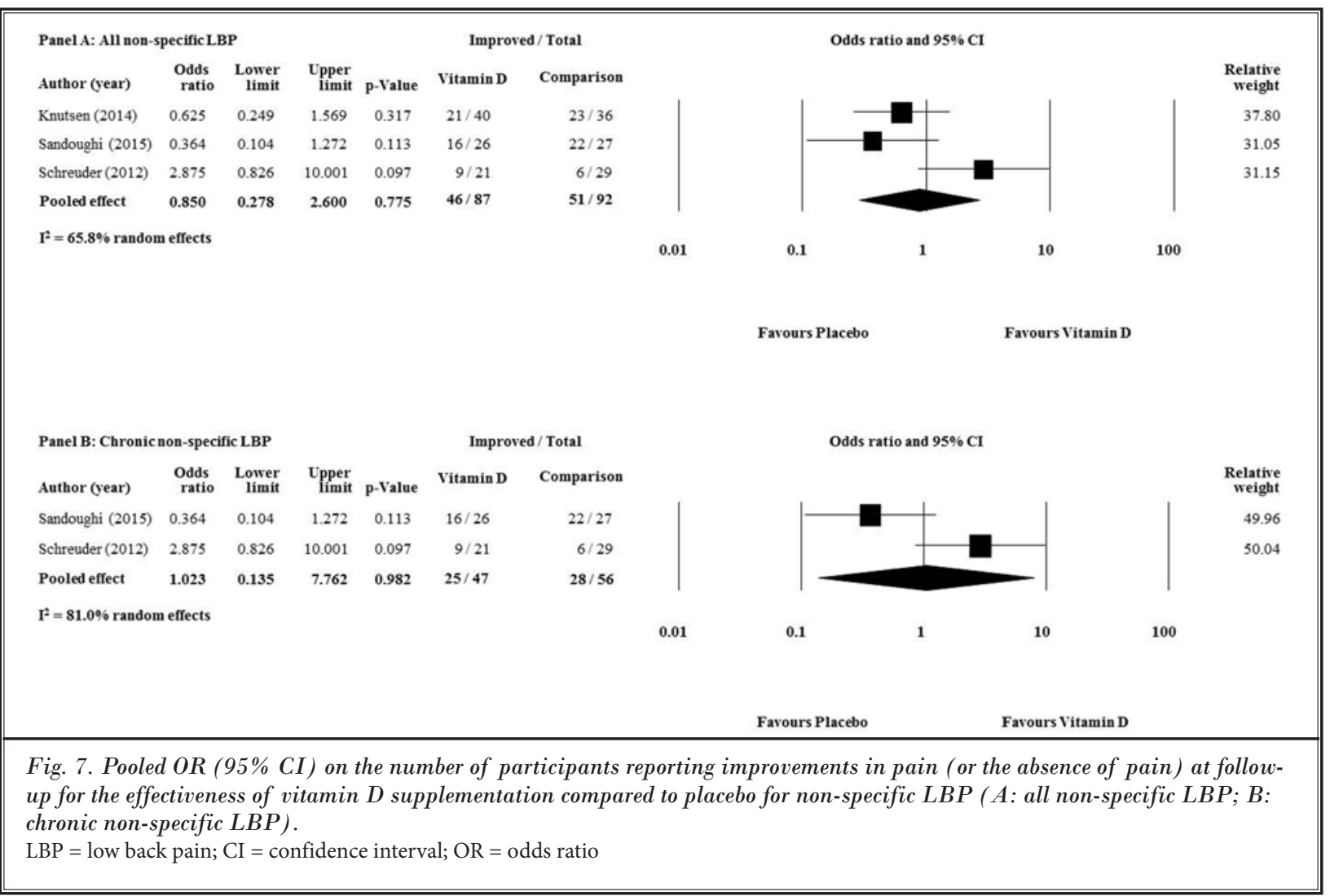

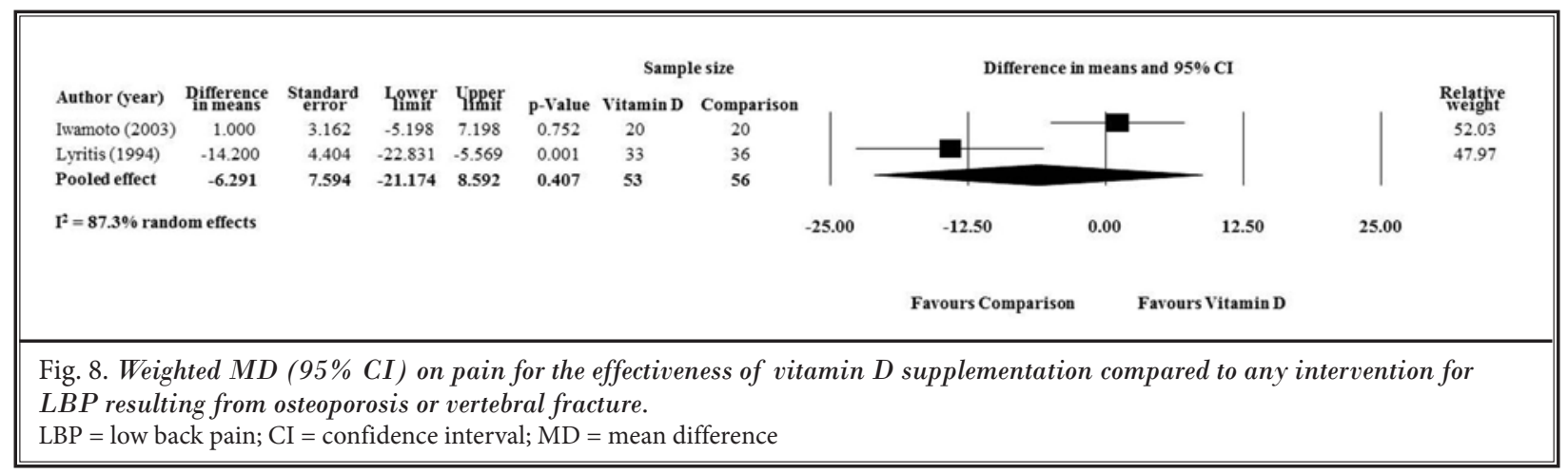

interventions) (MD $=-6.29,95 \% \mathrm{Cl}:-21.17$ to $8.59, P=$ $0.407, \mathrm{n}=2$ ) (Fig. 8) or in one study which compared alfacalcidol to a control group (6 months [0-10 scale]: $\mathrm{MD}=-0.10,95 \% \mathrm{Cl}:-0.66$ to $0.46, P=0.725,12$ months: $\mathrm{MD}=0.10,95 \% \mathrm{Cl}:-0.52$ to $0.72, P=0.752$ ) (45). When effectiveness was based on the number of participants reporting improvements in pain from baseline, there was no difference between alfacalcidol/calcitriol and any intervention (including placebo and other con- servative/pharmacological interventions) (pooled OR = 2.61, 95\% Cl: 0.04-160.59, $P=0.648, n=2$ ) (Fig. 9). One conference abstract showed that alfacalcidol was less effective than risedronate and eel calcitonin when pain was assessed by a VAS at 4 months $(P=0.0091$ and 0.0434 , respectively). However, the effect sizes were not reported and there was not sufficient data for inclusion in the meta-analyses (48). The only study which demonstrated that vitamin D supplementation was 


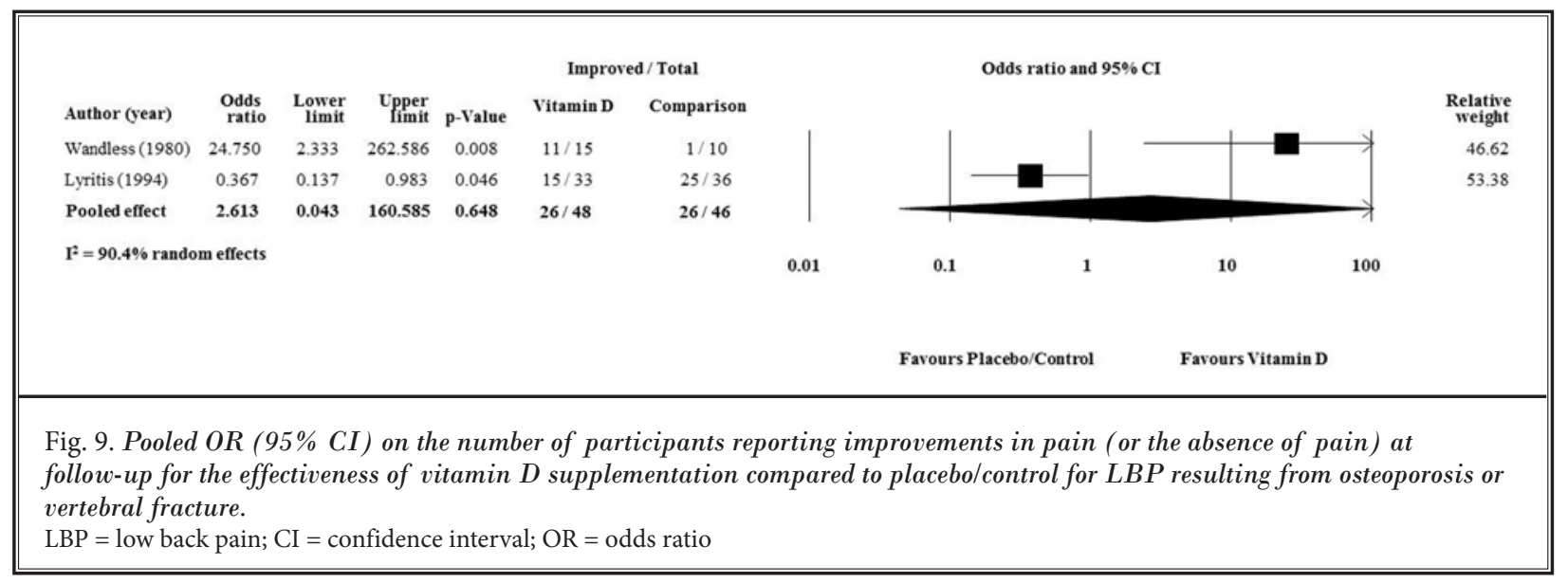

Table 3. Results from individual studies.

\begin{tabular}{|c|c|c|c|c|c|}
\hline $\begin{array}{c}\text { Author } \\
(\mathbf{y r})\end{array}$ & $\begin{array}{l}\text { Study } \\
\text { Design }\end{array}$ & Population & $\mathbf{n}$ & $\begin{array}{l}\text { Intervention vs. } \\
\text { Comparison }\end{array}$ & Results \\
\hline $\begin{array}{l}\text { Iwamoto } \\
\text { et al (2003) } \\
(45)\end{array}$ & RCT & $\begin{array}{l}\text { Postmenopausal } \\
\text { women between the } \\
\text { ages of } 60-86 \text { yrs } \\
\text { old with } \mathrm{O} P \text { and no } \\
\text { lumbar vertebral } \\
\text { fractures }\end{array}$ & 40 & $\begin{array}{l}1 \text { ug daily } \\
\text { alfacalcidol for vs. } \\
\text { control }\end{array}$ & $\begin{array}{l}\text { Face scale }(0-10) \\
6 \text { mos } \\
{ }^{*} \text { Alfacalcidol }(\mathrm{n}=20) \text { vs. control }(\mathrm{n}=20): \mathrm{MD}=-0.10 \text {, } \\
95 \% \text { CI: }-0.66 \text { to } 0.46, P=0.725 \\
12 \text { mos } \\
\text { Alfacalcidol }(\mathrm{n}=20) \text { vs. control }(\mathrm{n}=20): \\
\mathrm{MD}=0.10,95 \% \text { CI: }-0.52 \text { to } 0.72, P=0.752\end{array}$ \\
\hline $\begin{array}{l}\text { Knutsen et } \\
\text { al (2014) } \\
(46)\end{array}$ & RCT & $\begin{array}{l}\text { Healthy population } \\
\text { of men and women } \\
\text { aged between } 18-50 \\
\text { yrs old }\end{array}$ & 76 & $\begin{array}{l}25 \text { ug OR } 10 \text { ug } \\
\text { vitamin D3 daily } \\
\text { vs. placebo }\end{array}$ & $\begin{array}{l}16 \text { wks } \\
\text { VAS }(0-100) \\
\left.{ }^{*} 25 \text { ug vitamin D3 ( } \mathrm{n}=40\right) \text { vs. placebo }(\mathrm{n}=36) \text { : } \\
\mathrm{MD}=3.00,95 \% \mathrm{CI}--9.16 \text { to } 15.16, P=0.629 \\
10 \text { ug vitamin } \mathrm{D} 3(\mathrm{n}=41) \text { vs. placebo }(\mathrm{n}=36) \text { : } \\
\mathrm{MD}=6.00,95 \% \mathrm{CI}:-19.15 \text { to } 7.15, P=0.367 \\
\text { Number not in pain }(\%) \\
{ }^{\star} 25 \text { ug vitamin } \mathrm{D} 3(\mathrm{n}=40): 21(52.5 \%) \\
\text { Placebo }(\mathrm{n}=36): 23(50.0 \%) \\
\text { OR }=0.63,95 \% \text { CI: } 0.25 \text { to } 1.57, P=0.317 \\
10 \text { ug vitamin D3 }(\mathrm{n}=41): 18(43.9 \%) \\
\text { Placebo }(\mathrm{n}=36): 23(50.0 \%) \\
\text { OR }=0.44,95 \% \text { CI: } 0.18 \text { to } 1.11, P=0.082\end{array}$ \\
\hline $\begin{array}{l}\text { Sandoughi } \\
\text { et al (2015) } \\
(25)\end{array}$ & RCT & $\begin{array}{l}\text { Healthy population } \\
\text { of men and women } \\
\text { aged between } 18-40 \\
\text { yrs old }\end{array}$ & 53 & $\begin{array}{l}50000 \text { IU oral } \\
\text { vitamin D once per } \\
\text { wk vs. placebo }\end{array}$ & $\begin{array}{l}8 \text { wks } \\
\text { VAS }(0-10) \\
{ }^{*} \text { Vitamin } \mathrm{D} 3(\mathrm{n}=26) \text { vs. placebo }(\mathrm{n}=27) \text { : } \\
\mathrm{MD}=0.30,95 \% \text { CI: }-1.54 \text { to } 2.14, P=0.750 \\
\text { Improved from baseline }(\%) \\
{ }^{*} \text { Vitamin D3 }(\mathrm{n}=26): 16(61.5 \%) \\
\text { Placebo }(\mathrm{n}=27): 22(81.5 \%) \\
\text { OR }=0.36,95 \% \text { CI: } 0.10 \text { to } 1.27, P=0.113\end{array}$ \\
\hline $\begin{array}{l}\text { Wandless } \\
\text { et al (1980) } \\
(47)\end{array}$ & $\begin{array}{l}\text { Abstract of an } \\
\text { RCT }\end{array}$ & $\begin{array}{l}\text { Men and women with } \\
\text { a mean age (SD) of } \\
69.9 \text { (9.8) yrs with } \mathrm{OP} \\
\text { (some had vertebral } \\
\text { compression } \\
\text { fractures) }\end{array}$ & 25 & $\begin{array}{l}0.5-1.0 \mathrm{mg} \text { of } \\
\text { either alfacalcidol } \\
\text { or calcitriol daily } \\
\text { vs. placebo }\end{array}$ & $\begin{array}{l}\text { Improved from baseline }(\%) \\
6 \text { mos } \\
\text { *Alfacalcidol }(\mathrm{n}=15): 11(73.3 \%) \\
\text { Placebo }(\mathrm{n}=10): 1(10.0 \%) \\
\text { OR }=24.75,95 \% \text { CI: } 2.33 \text { to } 262.59, P=0.008\end{array}$ \\
\hline
\end{tabular}


Table 3 (cont.). Results from individual studies.

\begin{tabular}{|c|c|c|c|c|c|}
\hline $\begin{array}{c}\text { Author } \\
(\mathrm{yr})\end{array}$ & $\begin{array}{l}\text { Study } \\
\text { Design }\end{array}$ & Population & $\mathbf{n}$ & $\begin{array}{l}\text { Intervention vs. } \\
\text { Comparison }\end{array}$ & Results \\
\hline $\begin{array}{l}\text { Ota \& Ito } \\
(2007)(48)\end{array}$ & $\begin{array}{l}\text { Abstract of an } \\
\text { RCT }\end{array}$ & $\begin{array}{l}\text { Postmenopausal } \\
\text { women with BMD } \\
\text { below the young } \\
\text { adult mean and } \\
\text { without recent } \\
\text { vertebral fractures }\end{array}$ & 140 & $\begin{array}{l}1 \text { ug oral } \\
\text { alfacalcidol daily } \\
\text { vs. } \\
5 \text { mg oral } \\
\text { risedronate daily } \\
\text { OR } \\
60 \text { mg oral } \\
\text { raloxifene daily } \\
\text { OR } \\
20 \text { IU } \\
\text { intramuscular eel } \\
\text { calcitonin once } \\
\text { a wk }\end{array}$ & $\begin{array}{l}\text { VAS }(0-100) \\
1 \text { mo } \\
\text { Risedronate }(\mathrm{n}=35) \text { more effective than alfacalcidol }(\mathrm{n}= \\
\text { 35) }(P=0.0035) \\
4 \text { mos } \\
\text { Risedronate }(\mathrm{n}=35) \text { more effective than alfacalcidol }(\mathrm{n}= \\
\text { 35) }(P=0.0091) \\
\text { Eel calcitonin }(\mathrm{n}=35) \text { more effective than alfacalcidol }(\mathrm{n}= \\
\text { 35) }(P=0.0434)\end{array}$ \\
\hline $\begin{array}{l}\text { Majima et } \\
\text { al (2009) } \\
(50)\end{array}$ & $\begin{array}{l}\text { Non- } \\
\text { randomized } \\
\text { controlled } \\
\text { trial }\end{array}$ & $\begin{array}{l}\text { Men recently } \\
\text { diagnosed with } \mathrm{OP} \\
\text { with mean age }(\mathrm{SD}) \\
\text { of } 63.62(9.4) \text { yrs in } \\
\text { the vitamin } \mathrm{D} \text { group } \\
\text { and } 63.98(8.7) \text { yrs in } \\
\text { the risedronate group }\end{array}$ & 62 & $\begin{array}{l}1 \text { ug oral } \\
\text { alfacalcidol daily } \\
\text { vs. } 2.5 \text { mg oral } \\
\text { risedronate daily }\end{array}$ & $\begin{array}{l}\text { VAS }(0-100) \\
3 \text { mos } \\
{ }^{*} \text { Alfacalcidol }(\mathrm{n}=21) \text { vs. risedronate }(\mathrm{n}=41) \text { : } \\
\mathrm{MD}=-3.73,95 \% \text { CI: }-14.82 \text { to } 7.36, P=0.510 \\
12 \text { mos } \\
\text { Alfacalcidol }(\mathrm{n}=21) \text { vs. risedronate }(\mathrm{n}=41) \text { : } \\
\mathrm{MD}=-5.86,95 \% \text { CI: }-17.51 \text { to } 5.79, P=0.324 \\
2 \text { yrs } \\
\text { Alfacalcidol }(\mathrm{n}=21) \text { vs. risedronate }(\mathrm{n}=41) \text { : } \\
\mathrm{MD}=-6.84,95 \% \text { CI: }-17.66 \text { to } 3.98, P=0.215\end{array}$ \\
\hline $\begin{array}{l}\text { Schreuder } \\
\text { et al (2012) } \\
(49)\end{array}$ & $\begin{array}{l}\text { Randomized } \\
\text { cross-over } \\
\text { trial }\end{array}$ & $\begin{array}{l}\text { Men and women } \\
\text { aged between } 18-60 \\
\text { yrs with vitamin } \\
D \text { deficiency }(<50 \\
\mathrm{nm} / \mathrm{L}) \text { and vising } \\
\text { their GP for recurrent } \\
\text { MS pain lasting }> \\
3 \text { mos }\end{array}$ & 50 & $\begin{array}{l}150,000 \text { IU single } \\
\text { dose of oral } \\
\text { vitamin D vs. } \\
\text { placebo }\end{array}$ & $\begin{array}{l}6 \text { wks } \\
\text { VAS }(0-100) \\
{ }^{*} \text { Vitamin } \mathrm{D}(\mathrm{n}=21) \text { vs. placebo }(\mathrm{n}=29): \\
\mathrm{MD}=-2.00,95 \% \text { CI: }-21.09 \text { to } 17.09, P=0.837 \\
\text { Improved from baseline }(\%) \\
{ }^{*} \text { Vitamin } \mathrm{D} 3(\mathrm{n}=21): 9(42.9 \%) \\
\text { Placebo }(\mathrm{n}=29): 6(20.7 \%) \\
\text { OR }=2.88,95 \% \text { CI: } 0.83 \text { to } 10.00, P=0.097\end{array}$ \\
\hline $\begin{array}{l}\text { Lyritis et al } \\
\text { (1994) (44) }\end{array}$ & RCT & $\begin{array}{l}\text { Postmenopausal } \\
\text { women with } \\
\text { established } \mathrm{OP}(>10 \\
\text { yrs) and at least } \\
\text { one non-traumatic } \\
\text { vertebral collapse; } \\
\text { mean age (SD) of } \\
66.3(8.5) \text { yrs in the } \\
\text { intervention group } \\
\text { and } 67.5 \text { (9.1) yrs } \\
\text { in the comparison } \\
\text { group }\end{array}$ & 88 & $\begin{array}{l}1 \text { ug oral } \\
\text { alfacalcidol daily } \\
\text { (plus placebo } \\
\text { intramuscular } \\
\text { injections) vs. } 50 \\
\text { mg intramuscular } \\
\text { injections of } \\
\text { nandrolone } \\
\text { decanoate every } \\
3 \text { wks }\end{array}$ & $\begin{array}{l}6 \text { mos } \\
5 \text {-point pain scale } \\
{ }^{\star A l f a c a l c i d o l}(\mathrm{n}=37) \text { vs. nandrolone decanoate }(\mathrm{n}=40) \text { : } \\
\mathrm{MD}=-0.21,95 \% \text { CI: }-0.57 \text { to } 0.15, P=0.252 \\
12 \text { mos } \\
5 \text {-point pain scale } \\
\text { Alfacalcidol }(\mathrm{n}=33) \text { vs. nandrolone decanoate }(\mathrm{n}=36) \text { : } \\
\mathrm{MD}=-0.71,95 \% \text { CI: }-1.14 \text { to }-0.28, P=0.001 \\
\text { Improved from baseline }(\%) \text { at } \\
{ }^{\star} \text { Alfacalcidol }(\mathrm{n}=33): 15(45.5 \%) \\
\text { nandrolone decanoate }(\mathrm{n}=36): 25(69.4 \%) \\
\text { OR }=0.37,95 \% \text { CI: } 0.45 \text { to } 1.31, P=0.327\end{array}$ \\
\hline
\end{tabular}

$\mathrm{n}=$ number of participants that entered the analyses; $\mathrm{LBP}=$ low back pain; VAS = visual analog scale; $\mathrm{RCT}=$ randomized controlled trial; $\mathrm{SD}=$ standard deviation; $\mathrm{BMD}$ = bone mineral density; $\mathrm{MS}$ = musculoskeletal; GP = general practitioner; $\mathrm{OP}$ = osteoporosis; $\mathrm{IU}$ = international units; $\mathrm{ug}=$ micrograms; $\mathrm{mg}=$ milligrams; $\mathrm{nm}=$ nanomole

(a) $=1 \mathrm{IU}$ of vitamin D3 $=0.025 \mathrm{ug}=0.000025 \mathrm{mg}$

${ }^{*}$ Results included in meta-analysis

effective for LBP was reported in a conference abstract and included 25 participants with a mean age (SD) of 69.9 (9.8) years old, diagnosed with osteoporosis (selfreported reduction in pain at 6 months: $O R=24.75$, $95 \% \mathrm{Cl}: 2.33$ to $262.59, P=0.008$ ) (47) (Table 3).

\section{Adverse Events}

Only 4 of the 8 included trials reported on the incidence of adverse events $(45,46,48,49)$, although no study presented objective data. Three of these studies stated there were no adverse effects of vitamin D 
supplementation $(48,49)$, including issues related to the following areas: gastrointestinal, skin, nervous system, musculoskeletal, or urinary-tract (45). One study stated that adverse events were few, mild, and equally distributed between vitamin $\mathrm{D}$ and placebo groups at all time-points (46).

\section{Discussion}

This is the first systematic review to investigate the effect of vitamin D supplementation for numerous presentations of LBP, such as non-specific LBP and LBP resulting from osteoporosis or vertebral fractures, and it may inform whether the benefits of vitamin $D$ supplementation is dependent on an individual's clinical presentation. Vitamin D has the potential to reduce pain and inflammation by modulating sensory neuron excitability $(52,53)$ and the presence of anti- and proinflammatory cytokines $(30,54-56)$. In addition, higher vitamin $D$ levels have been linked to increases in muscle strength $(57,58)$, providing rationale for how vitamin D supplementation may improve pain and function in individuals with LBP. However, this review found very low quality evidence that vitamin $D$ supplementation is more effective than placebo, no intervention, or other conservative/pharmacological interventions for LBP, regardless of the type of LBP (non-specific LBP or LBP due to osteoporosis or vertebral fractures) or the type of vitamin D supplementation (vitamin D3 or alfacalcidol/ calcitriol).

\section{Comparison to Previous Studies}

This review highlights that adequately powered and well-designed clinical trials investigating vitamin D supplementation for LBP are mostly missing from the literature, resulting in very low quality evidence overall. Despite numerous studies reporting the beneficial effects of vitamin D supplementation for LBP, many could not be included in this review as they failed to investigate an appropriate comparison (e.g., no intervention or placebo) (59) or combined vitamin D supplementation with other active therapies (e.g., calcium supplementation) (60) (Fig. 1). Of the 8 clinical trials included in this review, only one scored $\geq 7$ on the PEDro scale $(25,46)$, with the most common methodological limitations being a lack of therapist or assessor blinding, no intention-to-treat analysis, or lack of allocation concealment. Despite this, the results from the highest quality study (PEDro score $=8$ ) $(46)$ with the largest sample size $(\mathrm{n}=76)(16$ weeks $[0-100$ scale]: $\mathrm{MD}=3.00,95 \% \mathrm{Cl}$ : -9.16 to $15.16, P=0.629$ ) (Table 3 ) were still in line with the overall results of this study, showing that vitamin D supplementation is no more effective than placebo for reducing pain levels in individuals with LBP (Fig. 3 \& 4). Despite these negative findings, the effect of vitamin D supplementation on LBP remains a topic of interest. This is likely driven by a desire to discover an optimal type or dosage of vitamin D supplementation that will elicit the greatest response or a specific sub-group of individuals who will respond favorably to vitamin $D$ supplementation (e.g., individuals with clinical and radiographic evidence of disc herniation (26) or with a particular level of vitamin $D$ deficiency).

There is evidence to support the choice of the type of vitamin D supplementation (e.g., vitamin D3 vs. alfacalcidol) for decreasing mortality in older adults (61) and for reducing the incidence of fractures and rate of falls in individuals with osteoporosis (62), while other studies suggest additional benefits of higher doses of vitamin $D$ supplementation compared to lower doses for various diseases $(63,64)$. Similarly, the results of our review may suggest the type and overall dose of vitamin D supplementation ('daily dose' vs. 'intervention duration') can influence the response to treatment in individuals with LBP. However, the effective dosage of vitamin $D$ for LBP needs to be viewed in light of safety recommendations. The Institute of Medicine recommends $10 \mathrm{ug}$ vitamin D3 daily for both adults and children (20 ug for those over 70 years old), with a tolerable upper intake level at 100 ug daily (65), while the Endocrine Practice Guidelines Committee recommends 37.5-50 ug vitamin D3 daily for people at risk of vitamin $D$ deficiency $(<20 \mathrm{ng} / \mathrm{mL})$, with a tolerable upper intake level at 200 ug daily (66). Furthermore, other guidelines recommend taking 165 ug daily for 8-12 weeks to reach sufficient vitamin $D$ levels, then taking a maintenance dose of 75 ug daily to prevent the recurrence of vitamin $D$ deficiency while avoiding toxicity (67). In contrast, there are no guidelines for the use of vitamin $\mathrm{D}$ analogues, such as alfacalcidol or calcitriol, as these medications are usually prescribed by a medical professional. However, a report from the Medicines and Healthcare products Regulatory Agency recommends a safe dosage of 1-3 ug alfacalcidol per day for adults, with higher doses (3-5 ug/day) recommended for the treatment of severe hypocalcaemia (68). The only study (conference abstract) that demonstrated a beneficial effect of vitamin $D$ supplementation compared to placebo prescribed 500-1000 ug alfacalcidol or calcitriol daily for 6 months (7 participants received calcitriol and 8 received alfacalcidol but were included in the same 
group) in older individuals (mean age [SD]: 69.9 [9.8]) with LBP due to osteoporosis or vertebral fractures (47) and found a significant between-group difference in the number of individuals who reported reductions in pain from baseline (OR $=24.75,95 \% \mathrm{Cl}: 2.33$ to 262.59 , $P=0.008$ ) (Table 3). However, this dosage is well above the Medicines and Healthcare products Regulatory Agency recommendations (68), which brings into question the safety of this dose and whether the dosage reported in this conference abstract was correct. Nevertheless, a lower overall dosage of alfacalcidol (1 ug daily for 12 months) was not more effective than no intervention (12 months [0-10 pain scale]: $M D=0.10$, $95 \%$ Cl: -0.52 to $0.72, P=0.752$ ) (45) or other conservative/pharmacological interventions for individuals with LBP resulting from osteoporosis or vertebral fractures $(44,48,50)$. These findings may suggest a higher overall dose of alfacalcidol or calcitriol is necessary to provide a beneficial effect in individuals with LBP resulting from osteoporosis or vertebral fractures. However, we cannot rule out the possibility that the prescription of calcitriol for some participants in the study with the highest dosage influenced these results (47). On the other hand, the pooled results from the 3 studies prescribing vitamin D3 for non-specific LBP (dosage ranging from 25-179 ug daily and intervention duration ranging from 6-16 weeks) failed to show a beneficial effect on pain intensity compared to a placebo (weighted $\mathrm{MD}=$ $1.90,95 \% \mathrm{Cl}:-7.06$ to $10.86, P=0.678, \mathrm{n}=3$ ) (Fig. 6 ). Since the studies prescribing higher doses of vitamin D3 were generally shorter in duration, it was not possible to investigate the overall impact of vitamin D3 dosage on treatment outcomes. Despite this, one randomized controlled trial included in our review (49) prescribed an overall dosage (179 ug vitamin D3 daily for 6 weeks) close to the tolerable upper intake levels but failed to demonstrate a beneficial effect for people with nonspecific LBP. Therefore, if research continues to investigate vitamin D3 supplementation for non-specific LBP, it may be more important to consider the target population rather than the overall dosage to build on these results rather than replicate them.

For over a decade, the search for a particular subgroup of individuals who demonstrate a more favorable response to an intervention has been a popular topic in the field of LBP (69). However, the findings of this review suggest that the effect of vitamin $D$ supplementation is no different for individuals with non-specific LBP or LBP resulting from osteoporosis or vertebral fractures, despite one study suggesting higher doses of alfacal- cidol/calcitriol were effective for individuals with LBP resulting from osteoporosis or vertebral fractures (47). Future studies exploring sub-groups of individuals likely to respond to vitamin $D$ supplementation should have a clear rationale, since implementing a well-designed and adequately powered sub-group study requires a large amount of planning and resources $(69,70)$. Although this review failed to clearly identify a sub-group of participants who respond to vitamin D supplementation, evidence from existing observational studies that investigate which populations with LBP have the greatest degree of vitamin $D$ deficiency could help to identify individuals who demonstrate a favorable response to vitamin $\mathrm{D}$ supplementation. There are numerous studies providing evidence for a greater degree of vitamin $D$ deficiency in younger women with LBP ( $<50$ years old) (71-74), while evidence for the association between vitamin D levels and LBP in older men and women ( $>60$ years old) appears to be conflicting (75-77). However, these findings may be explained by the geographical location of individual studies, since the studies reporting significant associations were all conducted in the Middle-East or India $(71,72,78,79)$. Nevertheless, these findings may point to a potential benefit of vitamin $D$ supplementation in younger women with LBP. Finally, an additional consideration for future research investigating vitamin $D$ supplementation for $L B P$ is the importance of measuring and reporting serum $25(\mathrm{OH})$ $D$ before and after supplementation to better understand the mechanism of effect. Only one study included in this review used vitamin $D$ deficiency $(<20 \mathrm{ng} / \mathrm{mL})$ as part of their inclusion criteria (49), while 2 studies reported mean serum $25(\mathrm{OH})$ D concentrations $<20 \mathrm{ng} / \mathrm{mL}$ at baseline for their sample $(25,46)$. This information is important since the size of the effect may be dependent on the presence of vitamin $D$ deficiency prior to supplementation. In addition, only 2 studies reported serum 25(OH)D concentrations following treatment $(25,46)$ and showed that serum $25(\mathrm{OH}) \mathrm{D}$ concentrations reached normal levels following supplementation $(>20$ $\mathrm{ng} / \mathrm{mL}$ ). However, these improvements did not correlate with improvements in symptoms of pain.

\section{Strengths and Limitations}

Our study has a number of strengths. First, we were able to include 8 clinical trials in this review. This is a substantial improvement on the only other published systematic review on vitamin D and LBP, which only included one clinical trial (80). Second, pooling the results from 5 randomized controlled trials allowed us 
to quantify the effect of vitamin $D$ supplementation on LBP, while numerous sub-group analyses explored whether this effect was influenced by the presentation of LBP (e.g., non-specific LBP or LBP due to osteoporosis or vertebral fractures) or type of vitamin $D$ supplementation prescribed (e.g., vitamin D3 or alfacalcidol).

This study had a number of limitations which need to be considered. First, including any clinical trial that provided a comparison between individuals receiving some form of vitamin D supplementation and a comparison treatment, including no intervention, might introduce substantial between-study heterogeneity. In addition, including studies reported in the form of conference abstracts makes it hard to judge the overall quality of evidence, as information about study design and implementation is limited. However, due to a small number of eligible studies ( $n=8$ ), keeping a broad inclusion criteria and including conference abstracts gives a better overall picture of the evidence and reduces the risk of publication bias (81). Furthermore, only 2 studies included in this review were conference abstracts, and our main findings were derived from meta-analyses where conference abstracts were excluded (Fig. 2 \& 3). Second, one of the conference abstracts included 140 participants but failed to report effect sizes. This meant a comparison between participant characteristics (e.g., age, gender, type of LBP, etc.) and effect sizes was not possible, and we could not include this study in any meta-analysis. Third, 2 studies allowed participants in the vitamin $D$ and placebo groups to take analgesics as required $(25,49)$. This could mask the true effect of vitamin $D$ supplementation if the utilization of analgesics was significantly different between groups. However, both studies outlined that there were no significant between-group differences in self-reported analgesic use, with one study reporting objective data (25). Finally, the overall quality of evidence was "very low." This was predominately due to the low methodological quality of the included studies (with $78.3 \%$ of the trials, weighted by their sample size, scoring $<7$ on the PEDro scale), and small sample sizes. Despite this, the results of our review provide an overall picture of the effect of vitamin D supplementation for LBP. Researchers interested in continuing to explore this topic should consider the current quality of the evidence and ensure they implement well-designed and adequately powered clinical trials to build on the evidence in this field.

\section{Conclusion}

This review found very low quality evidence that vitamin D supplementation is more effective than placebo, no intervention, or other conservative/pharmacological interventions for LBP, regardless of the type of LBP (non-specific or LBP due to osteoporosis or vertebral fractures) or vitamin D supplementation (vitamin D3 or alfacalcidol). Until well-designed and adequately powered clinical trials suggest otherwise, the prescription of vitamin D for LBP cannot be recommended. 


\section{Appendix 1. Search strategy.}

\section{MEDLINE}

\begin{tabular}{|c|c|}
\hline & Searches \\
\hline \multirow{20}{*}{ Vitamin D } & $\exp$ Vitamin D/ \\
\hline & “vitamin D".mp \\
\hline & “vitamin D2".mp \\
\hline & "vitamin D3".mp \\
\hline & "1-alpha hydroxyvitamin D3".mp \\
\hline & "1-alpha hydroxycalciferol".mp \\
\hline & "1,25 dihydroxyvitamin D3".mp \\
\hline & "1,25 dihydroxycholecalciferol".mp \\
\hline & “25 hydroxycholecalciferol".mp \\
\hline & “25 hydroxyvitamin D”.mp \\
\hline & "alfacalcidol".mp \\
\hline & “calcidiol".mp \\
\hline & “calcitriol".mp \\
\hline & "calcifediol".mp \\
\hline & "calciferol".mp \\
\hline & “ergocalciferol".mp \\
\hline & exp Ergocalciferols/ \\
\hline & "cholecalciferol".mp \\
\hline & exp Cholecalciferol/ \\
\hline & 1 or 2 or 3 or 4 or 5 or 6 or 7 or 8 or 9 or 10 or 11 or 12 or 13 or 14 or 15 or 16 or 17 or 18 or 19 \\
\hline \multirow{17}{*}{$\begin{array}{c}\text { Low Back } \\
\text { Pain }\end{array}$} & exp Back Pain/ \\
\hline & "back pain".mp \\
\hline & "backpain”.mp \\
\hline & exp Low Back Pain/ \\
\hline & "low back pain".mp \\
\hline & "backache".mp \\
\hline & "back ache".mp \\
\hline & (lumbar adj5 pain).ti,ab \\
\hline & "lumbar pain".mp \\
\hline & "spinal pain".mp \\
\hline & "lumbago".mp. \\
\hline & "lower back pain".mp \\
\hline & “dorsalgia".mp \\
\hline & “vertebral pain”.mp \\
\hline & 21 or 22 or 23 or 24 or 25 or 26 or 27 or 28 or 29 or 30 or 31 or 32 or 33 or 34 \\
\hline & 20 and 35 \\
\hline & Limit 36 to humans \\
\hline
\end{tabular}


Is Vitamin D Supplementation Effective for Low Back Pain?

\section{CINHAL}

\begin{tabular}{|c|c|}
\hline & Searches \\
\hline \multirow{21}{*}{ Vitamin D } & MH "Vitamin D+" \\
\hline & MH “Vitamin D Deficiency+" \\
\hline & MH "Ergocalciferols" \\
\hline & MH "Cholecalciferol” \\
\hline & "vitamin D" \\
\hline & "vitamin D2" \\
\hline & "vitamin D3" \\
\hline & "1-alpha hydroxyvitamin D3" \\
\hline & "1,25 dihydroxyvitamin D3" \\
\hline & "1,25 dihydroxycholecalciferol" \\
\hline & "25-hydroxycholecalciferol" \\
\hline & "25 hydroxycholecalciferol" \\
\hline & "25 hydroxyvitamin D" \\
\hline & "25-hydroxy-vitamin D" \\
\hline & "alfacalcidol" \\
\hline & "calcidiol" \\
\hline & "calcitriol" \\
\hline & MH “Calcitriol” \\
\hline & "calcifediol" \\
\hline & "calciferol" \\
\hline & 1 or 2 or 3 or 4 or 5 or 6 or 7 or 8 or 9 or 10 or 11 or 12 or 13 or 14 or 15 or 16 or 17 or 18 or 19 or 20 \\
\hline \multirow{14}{*}{ Low back pain } & (MH “Back Pain+") \\
\hline & "back pain" \\
\hline & (MH "Low Back Pain") \\
\hline & "low back pain" \\
\hline & "lumbago" \\
\hline & "backache" \\
\hline & "back ache" \\
\hline & "lumbar pain" \\
\hline & "spinal pain" \\
\hline & "backpain" \\
\hline & "lower back pain" \\
\hline & "dorsalgia" \\
\hline & "vertebral pain" \\
\hline & 22 or 23 or 23 or 24 or 25 or 26 or 27 or 28 or 29 or 30 or 31 or 32 or 33 or 34 \\
\hline
\end{tabular}


Pain Physician: March/April 2018; 21:121-145

EMBASE

\begin{tabular}{|c|c|}
\hline & Searches \\
\hline \multirow{18}{*}{ Vitamin D } & exp vitamin $\mathrm{D} /$ \\
\hline & “vitamin D”.mp \\
\hline & “vitamin D2".mp \\
\hline & "vitamin D3".mp \\
\hline & “1-alpha hydroxyvitamin D3".mp \\
\hline & “1-alpha-hydroxy-calciferol”.mp \\
\hline & “1,25 dihydroxyvitamin D3".mp \\
\hline & "1,25 dihydroxycholecalciferol".mp \\
\hline & " 25 hydroxycholecalciferol".mp \\
\hline & “25 hydroxyvitamin D”.mp \\
\hline & "alfacalcidol".mp \\
\hline & “calcidiol”.mp \\
\hline & “calcitriol".mp \\
\hline & "calcifediol".mp \\
\hline & “calciferol".mp \\
\hline & “ergocalciferol".mp \\
\hline & “cholecalciferol".mp \\
\hline & 1 or 2 or 3 or 4 or 5 or 6 or 7 or 8 or 9 or 10 or 11 or 12 or 13 or 14 or 15 or 16 or 17 \\
\hline \multirow{16}{*}{ Low back pain } & exp Backache/ \\
\hline & "backache".mp \\
\hline & "back ache".mp \\
\hline & exp Low back pain/ \\
\hline & "low back pain".mp \\
\hline & exp Spinal pain/ \\
\hline & "spinal pain".mp \\
\hline & "back pain".mp \\
\hline & "lumbago".mp \\
\hline & "lumbar pain".mp \\
\hline & "lower back pain".mp \\
\hline & “vertebral pain”.mp \\
\hline & “dorsalgia".mp \\
\hline & 19 or 20 or 21 or 22 or 23 or 24 or 25 or 26 or 27 or 28 or 29 or 30 or 31 \\
\hline & 18 and 32 \\
\hline & Limit 33 to humans \\
\hline
\end{tabular}


Is Vitamin D Supplementation Effective for Low Back Pain?

\section{AMED}

\begin{tabular}{|c|c|}
\hline Searches & \\
\hline \multirow{16}{*}{ Vitamin D } & $\exp$ Vitamin d/ \\
\hline & “vitamin D”.mp \\
\hline & "vitamin D2".mp \\
\hline & “vitamin D3".mp \\
\hline & “1,25 dihydroxyvitamin D3”.mp \\
\hline & “25 hydroxycholecalciferol”.mp \\
\hline & “25 hydroxyvitamin D”.mp \\
\hline & “alfacalcidol”.mp \\
\hline & “calcidiol”.mp \\
\hline & “calcitriol”.mp \\
\hline & “calcifediol".mp \\
\hline & “calciferol”.mp \\
\hline & “ergocalciferol".mp \\
\hline & “cholecalciferol”.mp \\
\hline & exp Cholecalciferols/ \\
\hline & 1 or 2 or 3 or 4 or 5 or 6 or 7 or 8 or 9 or 10 or 11 or 12 or 13 or 14 or 15 \\
\hline \multirow{16}{*}{ Low back pain } & exp Low Back Pain/ \\
\hline & exp Backache/ \\
\hline & "low back pain".mp \\
\hline & "back pain".mp \\
\hline & "backpain”.mp \\
\hline & "backache".mp \\
\hline & "back ache".mp \\
\hline & (lumbar adj5 pain).ti,ab \\
\hline & "lumbar pain".mp \\
\hline & "spinal pain".mp \\
\hline & lumbago.mp \\
\hline & "lower back pain".mp \\
\hline & dorsalgia.mp \\
\hline & “vertebral pain”.mp \\
\hline & 17 or 18 or 19 or 20 or 21 or 22 or 23 or 24 or 25 or 26 or 27 or 28 or 29 or 30 or 31 or 32 \\
\hline & 16 and 31 \\
\hline
\end{tabular}




\section{Scopus}

\begin{tabular}{|c|c|}
\hline & Searches \\
\hline \multirow{24}{*}{ Vitamin D } & TITLE-ABS-KEY(“vitamin D”) \\
\hline & TITLE-ABS-KEY(“vitamin D2") \\
\hline & TITLE-ABS-KEY(“vitamin D3”) \\
\hline & TITLE-ABS-KEY(“1-alpha hydroxyvitamin D3”) \\
\hline & TITLE-ABS-KEY(“1-alpha-hydroxy-vitamin D3”) \\
\hline & TITLE-ABS-KEY(“1-alpha hydroxycalciferol”) \\
\hline & TITLE-ABS-KEY(“1-alpha-hydroxy-calciferol”) \\
\hline & TITLE-ABS-KEY(“1,25 dihydroxyvitamin D3”) \\
\hline & TITLE-ABS-KEY(“1,25-dihydroxy-vitamin D3”) \\
\hline & TITLE-ABS-KEY(“1,25 dihydroxycholecalciferol”) \\
\hline & TITLE-ABS-KEY(“1,25-dihydroxycholecalciferol”) \\
\hline & TITLE-ABS-KEY(25-hydroxycholecalciferol) \\
\hline & TITLE-ABS-KEY(“25 hydroxycholecalciferol”) \\
\hline & TITLE-ABS-KEY(“25 hydroxyvitamin D”) \\
\hline & TITLE-ABS-KEY(“25-hydroxy-vitamin D”) \\
\hline & TITLE-ABS-KEY(25-hydroxycholecalciferol) \\
\hline & TITLE-ABS-KEY(alfacalcidol) \\
\hline & TITLE-ABS-KEY(calcidiol) \\
\hline & TITLE-ABS-KEY(calcitriol) \\
\hline & TITLE-ABS-KEY(calcifediol) \\
\hline & TITLE-ABS-KEY(calciferol) \\
\hline & TITLE-ABS-KEY(ergocalciferol) \\
\hline & TITLE-ABS-KEY(cholecalciferol) \\
\hline & 1 or 2 or 3 or 4 or 5 or 6 or 7 or 8 or 9 or 10 or 11 or 12 or 13 or 14 or 15 or 16 or 17 or 18 or 19 or 20 or 21 or 22 or 23 \\
\hline \multirow{14}{*}{ Low back pain } & ALL(“back pain) \\
\hline & TITLE-ABS-KEY(backpain) \\
\hline & ALL(“low back pain”) \\
\hline & TITLE-ABS-KEY(backache) \\
\hline & TITLE-ABS-KEY(“back ache”) \\
\hline & TITLE-ABS-KEY(“lumbar pain”) \\
\hline & TITLE-ABS-KEY(“spinal pain”) \\
\hline & TITLE-ABS-KEY(lumbago) \\
\hline & TITLE-ABS-KEY(“lower back pain”) \\
\hline & TITLE-ABS-KEY(dorsalgia) \\
\hline & TITLE-ABS-KEY(“vertebral pain”) \\
\hline & 26 or 27 or 28 or 29 or 30 or 31 or 32 or 33 or 34 or 35 \\
\hline & 24 and 36 \\
\hline & Exclude: "animals" and "animal" \\
\hline
\end{tabular}


Is Vitamin D Supplementation Effective for Low Back Pain?

\section{Web of Science}

\begin{tabular}{|c|c|}
\hline & Searches \\
\hline \multirow{24}{*}{ Vitamin D } & TS = (“vitamin D”) \\
\hline & TS = (“vitamin D2") \\
\hline & TS = (“vitamin D3") \\
\hline & TS = (“1-alpha hydroxyvitamin D3”) \\
\hline & TS = ("1-alpha-hydroxy-vitamin D3") \\
\hline & TS = ("1-alpha hydroxycalciferol") \\
\hline & TS = ("1-alpha-hydroxy-calciferol") \\
\hline & TS $=(" 1,25$ dihydroxyvitamin D3") \\
\hline & TS = (“1,25-dihydroxy-vitamin D3") \\
\hline & TS = ("1,25 dihydroxycholecalciferol”) \\
\hline & TS = ("1,25-dihydroxycholecalciferol") \\
\hline & TS $=(25$-hydroxycholecalciferol $)$ \\
\hline & TS = ("25 hydroxycholecalciferol”) \\
\hline & TS = (“25 hydroxyvitamin D”) \\
\hline & TS = (“25-hydroxy-vitamin D”) \\
\hline & TS $=(25$-hydroxycholecalciferol $)$ \\
\hline & TS $=($ alfacalcidol $)$ \\
\hline & TS $=($ calcidiol $)$ \\
\hline & $\mathrm{TS}=($ calcitriol $)$ \\
\hline & $\mathrm{TS}=($ calcifediol $)$ \\
\hline & $\mathrm{TS}=($ calciferol $)$ \\
\hline & $\mathrm{TS}=($ ergocalciferol $)$ \\
\hline & $\mathrm{TS}=($ cholecalciferol $)$ \\
\hline & 1 or 2 or 3 or 4 or 5 or 6 or 7 or 8 or 9 or 10 or 11 or 12 or 13 or 14 or 15 or 16 or 17 or 18 or 19 or 20 or 21 or 22 or 23 \\
\hline \multirow{14}{*}{ Low back pain } & TS = (“back pain”) \\
\hline & TS $=($ backpain $)$ \\
\hline & $\mathrm{TS}=$ ("low back pain) \\
\hline & TS $=($ lumbago $)$ \\
\hline & TS $=$ (backache $)$ \\
\hline & TS = ("lumbar pain") \\
\hline & TS $=$ (“spinal pain") \\
\hline & TS = ("lower back pain") \\
\hline & $\mathrm{TS}=($ dorsalgia $)$ \\
\hline & TS $=$ ("vertebral pain") \\
\hline & 25 or 26 or 27 or 28 or 29 or 30 or 31 or 32 or 33 or 34 \\
\hline & 24 and 35 \\
\hline & $\mathrm{TS}=($ animals $)$ NOT TS $=$ (humans) \\
\hline & 36 NOT 37 \\
\hline
\end{tabular}




\section{References}

1. Vos T, Barber RM, Bell B, Bertozzi-Villa A, Biryukov S, Bolliger I, Charlson F, Davis A, Degenhardt L, Dicker D, Duan L, Erskine H, Feigin VL, Ferrari A), Fitzmaurice C, Fleming T, Graetz N, Guinovart C, Haagsma J, Hansen GM, Hanson SW, Heuton KR, Higashi $H$, Kassebaum N, Kyu H, Laurie E, Liang X, Lofgren K, Lozano R, Maclntyre MF, Moradi-Lakeh $M$, Naghavi $M$, Nguyen G, Odell S, Ortblad K, Roberts DA, Roth GA, Sandar L, Serina PT, Stanaway JD, Steiner C, Thomas B, Vollset SE, Whiteford H, Wolock TM, Ye P, Zhou M, Ãvila MA, Aasvang GM, Abbafati C, Ozgoren AA, Abd-Allah F, Aziz MIA, Abera SF, Aboyans V, Abraham JP, Abraham B, Abubakar I, Abu-Raddad LJ, Abu-Rmeileh NME, Aburto TC, Achoki T, Ackerman IN, Adelekan A, Ademi Z, Adou AK, Adsuar JC, Arnlov J, Agardh EE, Al Khabouri MJ, Alam SS, Alasfoor D, Albittar MI, Alegretti MA, Aleman AV, Alemu ZA, Alfonso-Cristancho R, Alhabib $S$, Ali $R$, Alla F, Allebeck P, Allen PJ, AlMazroa MA, Alsharif U, Alvarez E, Alvis-Guzman N, Ameli $\mathrm{O}$, Amini $\mathrm{H}$, Ammar W, Anderson $\mathrm{BO}$, Anderson HR, Antonio CAT, Anwari $\mathrm{P}$, Apfel $\mathrm{H}$, Arsenijevic VSA, Artaman A, Asghar RJ, Assadi R, Atkins LS, Atkinson C, Badawi A, Bahit MC, Bakfalouni T, Balakrishnan K, Balalla S, Banerjee A, Barker-Collo SL, Barquera S, Barregard L, Barrero LH, Basu S, Basu A, Baxter A, Beardsley J, Bedi N, Beghi E, Bekele T, Bell ML, Benjet C, Bennett DA, Bensenor IM, Benzian $\mathrm{H}$, Bernabe $\mathrm{E}$, Beyene TJ, Bhala N, Bhalla A, Bhutta Z, Bienhoff $K$, Bikbov $B$, Abdulhak $A B$, Blore JD, Blyth FM, Bohensky MA, Basara BB, Borges G, Bornstein NM, Bose D, Boufous $S$, Bourne RR, Boyers LN, Brainin $M$, Brauer $M$, Brayne CEG, Brazinova $A$, Breitborde NJK, Brenner $\mathrm{H}$, Briggs ADM, Brooks PM, Brown J, Brugha TS, Buchbinder R, Buckle GC, Bukhman G, Bulloch AG, Burch M, Burnett R, Cardenas R, Cabral NL, Nonato IRC, Campuzano JC, Carapetis JR, Carpenter DO, Caso V, Castaneda-Orjuela CA, CatalaLopez F, Chadha VK, Chang J-C, Chen $\mathrm{H}$, Chen W, Chiang PP, Chimed-Ochir $\mathrm{O}$, Chowdhury R, Christensen $\mathrm{H}$, Christophi CA, Chugh SS, Cirillo M, Coggeshall M, Cohen A, Colistro V, Colquhoun SM, Contreras AG, Cooper LT, Cooper C, Cooperrider K, Coresh J, Cortinovis $\mathrm{M}$, Criqui MH, Crump JA, Cuevas-Nasu L, Dandona R, Dandona L, Dansereau
E, Dantes HG, Dargan PI, Davey G, Davitoiu DV, Dayama A, De la Cruz-Gongora $\checkmark$, de la Vega SF, De Leo D, del PozoCruz B, Dellavalle RP, Deribe K, Derrett $S$, Des Jarlais DC, Dessalegn $M$, deVeber GA, Dharmaratne SD, Diaz-Torne C, Ding EL, Dokova K, Dorsey ER, Driscoll TR, Duber H, Durrani AM, Edmond KM, Ellenbogen RG, Endres M, Ermakov SP, Eshrati B, Esteghamati A, Estep K, Fahimi S, Farzadfar F, Fay DFJ, Felson DT, Fereshtehnejad S-M, Fernandes JG, Ferri CP, Flaxman A, Foigt N, Foreman KJ, Fowkes FGR, Franklin RC, Furst T, Futran ND, Gabbe BJ, Gankpe FG, Garcia-Guerra FA, Geleijnse JM, Gessner BD, Gibney KB, Gillum RF, Ginawi IA, Giroud M, Giussani G, Goenka S, Goginashvili K, Gona P, de Cosio TG, Gosselin RA, Gotay CC, Goto A, Gouda HN, Guerrant Rl, Gugnani HC, Gunnell D, Gupta R, Gupta R, Gutierrez RA, Hafezi-Nejad $N$, Hagan $H$, Halasa $Y$, Hamadeh RR, Hamavid H, Hammami M, Hankey G), Hao Y, Harb HL, Haro JM, Havmoeller R, Hay RJ, Hay S, Hedayati MT, Pi IBH, Heydarpour P, Hijar M, Hoek HW, Hoffman HJ, Hornberger JC, Hosgood HD, Hossain M, Hotez PJ, Hoy DG, Hsairi M, $\mathrm{Hu} \mathrm{H}, \mathrm{Hu}$ G, Huang JJ, Huang C, Huiart L, Husseini A, lannarone $M$, Iburg KM, Innos $\mathrm{K}$, Inoue $\mathrm{M}$, Jacobsen $\mathrm{KH}$, Jassal $S K$, Jeemon $P$, Jensen $P N$, Jha $V$, Jiang $G$, Jiang $Y$, Jonas JB, Joseph J, Juel K, Kan H, Karch A, Karimkhani C, Karthikeyan G, Katz R, Kaul A, Kawakami N, Kazi DS, Kemp AH, Kengne AP, Khader YS, Khalifa SEAH, Khan EA, Khan G, Khang Y-H, Khonelidze I, Kieling C, Kim D, Kim S, Kimokoti RW, Kinfu Y, Kinge JM, Kissela BM, Kivipelto M, Knibbs L, Knudsen AK, Kokubo Y, Kosen S, Kramer A, Kravchenko M, Krishnamurthi RV, Krishnaswami S, Defo BK, Bicer BK, Kuipers EJ, Kulkarni VS, Kumar K, Kumar GA, Kwan GF, Lai T, Lalloo R, Lam H, Lan Q, Lansingh VC, Larson $\mathrm{H}$, Larsson A, Lawrynowicz AEB, Leasher JL, Lee J-T, Leigh J, Leung R, Levi M, Li B, Li Y, Li Y, liang J, Lim S, Lin $\mathrm{H}-\mathrm{H}$, Lind $\mathrm{M}$, Lindsay MP, Lipshultz SE, Liu S, Lloyd BK, Ohno SL, Logroscino G, Looker KJ, Lopez AD, Lopez-Olmedo N, Lortet-Tieulent J, Lotufo PA, Low N, Lucas RM, Lunevicius R, Lyons RA, Ma J, Ma S, Mackay MT, Majdan M, Malekzadeh R, Mapoma CC, Marcenes W, March LM, Margono C, Marks GB, Marzan MB, Masci JR, Mason-Jones AJ, Matzopoulos RG, Mayosi BM, Mazorodze TT, McGill
NW, McGrath JJ, McKee M, McLain A, McMahon BJ, Meaney PA, Mehndiratta MM, Mejia-Rodriguez F, Mekonnen W, Melaku YA, Meltzer M, Memish ZA, Mensah G, Meretoja A, Mhimbira FA, Micha R, Miller TR, Mills EJ, Mitchell PB, Mock CN, Moffitt TE, Ibrahim NM, Mohammad KA, Mokdad AH, Mola GL, Monasta L, Montico M, Montine TJ, Moore AR, Moran AE, Morawska L, Mori R, Moschandreas J, Moturi WN, Moyer M, Mozaffarian D, Mueller UO, Mukaigawara M, Murdoch ME, Murray J, Murthy KS, Naghavi P, Nahas Z, Naheed A, Naidoo KS, Naldi L, Nand D, Nangia V, Narayan KMV, Nash D, Nejjari C, Neupane SP, Newman LM, Newton CR, Ng $M$, Ngalesoni FN, Nhung NT, Nisar MI, Nolte S, Norheim OF, Norman RE, Norrving $B$, Nyakarahuka L, Oh IH, Ohkubo T, Omer SB, Opio JN, Ortiz A, Pandian JD, Panelo CIA, Papachristou C, Park E-K, Parry CD, Caicedo AJP, Patten SB, Paul VK, Pavlin BI, Pearce N, Pedraza LS, Pellegrini CA, Pereira DM, Perez-Ruiz FP, Perico N, Pervaiz A, Pesudovs K, Peterson $C B$, Petzold M, Phillips MR, Phillips D, Phillips B, Piel FB, Plass D, Poenaru D, Polanczyk GV, Polinder S, Pope CA, Popova S, Poulton RG, Pourmalek F, Prabhakaran D, Prasad NM, Qato D, Quistberg DA, Rafay A, Rahimi K, Rahimi-Movaghar V, Rahman Su, Raju M, Rakovac I, Rana SM, Razavi H, Refaat A, Rehm J, Remuzzi G, Resnikoff S, Ribeiro AL, Riccio PM, Richardson L, Richardus $\mathrm{JH}$, Riederer AM, Robinson M, Roca A, Rodriguez A, Rojas-Rueda D, Ronfani L, Rothenbacher D, Roy N, Ruhago GM, Sabin N, Sacco RL, Ksoreide K, Saha S, Sahathevan R, Sahraian MA, Sampson U, Sanabria JR, Sanchez-Riera L, Santos IS, Satpathy M, Saunders JE, Sawhney M, Saylan MI, Scarborough P, Schoettker B, Schneider IJC, Schwebel DC, Scott JG, Seedat S, Sepanlou SG, Serdar B, Servan-Mori EE, Shackelford K, Shaheen A, Shahraz S, Levy TS, Shangguan S, She J, Sheikhbahaei S, Shepard DS, Shi P, Shibuya K, Shinohara Y, Shiri R, Shishani K, Shiue I, Shrime MG, Sigfusdottir ID, Silberberg DH, Simard EP, Sindi S, Singh JA, Singh L, Skirbekk V, Sliwa K, Soljak M, Soneji S, Soshnikov SS, Speyer $\mathrm{P}$, Sposato LA, Sreeramareddy CT, Stoeckl H, Stathopoulou VK, Steckling N, Stein MB, Stein DJ, Steiner TJ, Stewart A, Stork E, Stovner LJ, Stroumpoulis K, Sturua L, Sunguya BF, Swaroop M, 
Sykes BL, Tabb KM, Takahashi K, Tan F Tandon N, Tanne D, Tanner M, Tavakkoli M, Taylor HR, Te Ao BJ, Temesgen AM, Have MT, Tenkorang EY, Terkaw AS, Theadom AM, Thomas E, ThorneLyman AL, Thrift AG, Tleyjeh IM, Tonelli M, Topouzis F, Towbin JA, Toyoshima H, Traebert J, Tran BX, Trasande L, Trillini M, Truelsen T, Trujillo $U$, Tsilimbaris M, Tuzcu EM, Ukwaja KN, Undurraga EA, Uzun SB, van Brakel WH, van de Vijver $\mathrm{S}$, Dingenen RV, van Gool CH, Varakin YY, Vasankari TJ, Vavilala MS, Veerman LJ, Velasquez-Melendez G, Venketasubramanian $\mathrm{N}$, Vijayakumar L, Villalpando S, Violante FS, Vlassov VV, Waller S, Wallin MT, Wan X, Wang L, Wang J, Wang Y, Warouw TS, Weichenthal S, Weiderpass E, Weintraub RG, Werdecker A, Wessells KRR, Westerman R, Wilkinson JD, Williams HC, Williams TN, Woldeyohannes SM, Wolfe CDA, Wong JQ, Wong $\mathrm{H}$, Woolf AD, Wright JL, Wurtz B, Xu G, Yang G, Yano $Y$, Yenesew MA, Yentur GK, Yip P, Yonemoto N, Yoon S-J, Younis M, Yu C, Kim KY, Zaki MES, Zhang Y, Zhao Z, Zhao Y, Zhu J, Zonies D, Zunt JR, Salomon JA, Murray CJL. Global, regional, and national incidence, prevalence, and years lived with disability for 301 acute and chronic diseases and injuries in 188 countries, 1990-2013: A systematic analysis for the Global Burden of Disease Study 2013. The Lancet 2015; 386:743-800.

2. Walker BF, Muller R, Grant WD. Low back pain in Australian adults: The economic burden. Asia Pac J Public Health 2003; 15:79-87.

3. Wenig CM, Schmidt CO, Kohlmann T, Schweikert B. Costs of back pain in Germany. Eur J Pain 2009; 13:280-286.

4. Brunner F, Weiser S, Schmid A, Nordin M. Non-specific low back pain. In: Boos N, Aebi M (eds). Spinal Disorders: Fundamentals of Diagnosis and Treatment. Springer-Verlag Berlin Heidelberg, Berlin 2008, pp 585-601.

5. Brinjikji W, Luetmer PH, Comstock B, Bresnahan BW, Chen LE, Deyo RA, Halabi S, Turner JA, Avins AL, James K, Wald JT, Kallmes DF, Jarvik JG. Systematic literature review of imaging features of spinal degeneration in asymptomatic populations. AJNR Am J Neuroradiol 2015; 36:811-816.

6. Savage RA, Whitehouse GH, Roberts N. The relationship between the magnetic resonance imaging appearance of the lumbar spine and low back pain, age and occupation in males. Eur Spine J 1997; 6:106-114.
7. Henschke N, Maher CG, Refshauge KM, Herbert RD, Cumming RG, Bleasel J, York J, Das A, McAuley JH. Prevalence of and screening for serious spinal pathology in patients presenting to primary care settings with acute low back pain. Arthritis Rheum 2009; 60:3072-3080.

8. Henschke N, Maher CG, Refshauge KM. Screening for malignancy in low back pain patients: A systematic review. Eur Spine J 2007; 16:1673-1679.

9. Ettinger B, Black DM, Nevitt MC, Rundle AC, Cauley JA, Cummings SR, Genant HK. Contribution of vertebral deformities to chronic back pain and disability. The Study of Osteoporotic Fractures Research Group. J Bone Miner Res 1992; 7:449-456.

10. Koes BW, van Tulder M, Lin C-W, Macedo LG, McAuley J, Maher C. An updated overview of clinical guidelines for the management of non-specific low back pain in primary care. Eur Spine J 2010; 19:2075-2094.

11. van Middelkoop $M$, Rubinstein $S M$, Verhagen AP, Ostelo RW, Koes BW, van Tulder MW. Exercise therapy for chronic nonspecific low-back pain. Best Prac Res Clin Rheumatol 2010; 24:193-204.

12. Liddle SD, Gracey JH, Baxter GD. Advice for the management of low back pain: A systematic review of randomised controlled trials. Man Ther 2007; 12:310-327.

13. Goodwin VA, Hall AJ, Rogers E, Bethel A. Orthotics and taping in the management of vertebral fractures in people with osteoporosis: A systematic review. BMJ Open 2016; 6:e010657.

14. Giangregorio LM, Macintyre NJ, Thabane L, Skidmore CJ, Papaioannou A. Exercise for improving outcomes after osteoporotic vertebral fracture. Cochrane Database Syst Rev 2013; (1):CDoo8618.

15. An T, Hao J, Sun S, Li R, Yang M, Cheng G, Zou M. Efficacy of statins for osteoporosis: A systematic review and metaanalysis. Osteoporos Int 2017; 28:47-57.

16. Davis S, Martyn-St James M, Sanderson J, Stevens J, Goka E, Rawdin A, Sadler S, Wong R, Campbell F, Stevenson M, Strong M, Selby P, Gittoes N. A systematic review and economic evaluation of bisphosphonates for the prevention of fragility fractures. Health Technol Assess 2016; 20:1-406.

17. Body JJ, Bergmann P, Boonen S, Boutsen Y, Bruyere O, Devogelaer JP, Goemaere $S$, Hollevoet N, Kaufman JM, Milisen K, Rozenberg S, Reginster JY. Non-pharmacological management of osteoporosis: A consensus of the Bel- gian Bone Club. Osteoporos Int 2011; 22:2769-2788.

18. Saragiotto BT, Maher CG, Yamato TP, Costa LO, Costa LC, Ostelo RW, Macedo LG. Motor control exercise for nonspecific low back pain: A cochrane review. Spine (Phila Pa 1976) 2016; 41:1284-1295.

19. Ferreira ML, Ferreira PH, Latimer J, Herbert RD, Hodges PW, Jennings MD, Maher CG, Refshauge KM. Comparison of general exercise, motor control exercise and spinal manipulative therapy for chronic low back pain: A randomized trial. Pain 2007; 131:31-37.

20. Chou R, Deyo R, Friedly J, Skelly A, Hashimoto R, Weimer M, Fu R, Dana T, Kraegel P, Griffin J, Grusing S, Brodt E. Noninvasive treatments for low back pain. Rockville (MD): Agency for Healthcare Research and Quality (US) 2016; Report No.:16-EHCoO4-EF.

21. Newman M, Minns Lowe C, Barker K. Spinal orthoses for vertebral osteoporosis and osteoporotic vertebral fracture: A systematic review. Arch Phys Med Rehabil 2016; 97:1013-1025.

22. Stevenson $M$, Gomersall $\mathrm{T}$, Lloyd Jones M, Rawdin A, Hernández M, Dias S, Wilson $D$, Rees A. Percutaneous vertebroplasty and percutaneous balloon kyphoplasty for the treatment of osteoporotic vertebral fractures: A systematic review and cost-effectiveness analysis. Health Technol Assess 2014; 18:1-290.

23. Keller A, Hayden J, Bombardier C, van Tulder M. Effect sizes of non-surgical treatments of non-specific low-back pain. Eur Spine J 2007; 16:1776-1788.

24. Straube S, Derry S, Straube C, Moore RA. Vitamin $D$ for the treatment of chronic painful conditions in adults. Cochrane Database Syst Rev 2015; (5):CDoo7771.

25. Sandoughi M, Zakeri Z, Mirhosainee Z, Mohammadi M, Shahbakhsh S. The effect of vitamin $D$ on nonspecific low back pain. Int J Rheumat Dis 2015; 18:854-858.

26. Sedighi M, Haghnegahdar A. Role of vitamin $D_{3}$ in treatment of lumbar disc herniation--pain and sensory aspects: Study protocol for a randomized controlled trial. Trials 2014; 15:373.

27. Costa LCM, Maher CG, McAuley JH, Hancock MJ, Herbert RD, Refshauge KM, Henschke N. Prognosis for patients with chronic low back pain: Inception cohort study. BMJ 2009; 339:b3829.

28. Weaver CM, Gordon CM, Janz KF, Kalkwarf HJ, Lappe JM, Lewis R, O'Karma M, Wallace TC, Zemel BS. The National Osteoporosis Foundation's position state- 
ment on peak bone mass development and lifestyle factors: A systematic review and implementation recommendations. Osteoporos Int 2016; 27:1281-1386.

29. Cranney A, Horsley T, O'Donnell S, Weiler $\mathrm{H}$, Puil L, Ooi D, Atkinson S, Ward L, Moher D, Hanley D, Fang M, Yazdi F, Garritty C, Sampson M, Barrowman N, Tsertsvadze A, Mamaladze V. Effectiveness and safety of vitamin $D$ in relation to bone health. Evid Rep Technol Assess (Full Rep) 2007; (158):1-235.

30. Calton EK, Keane KN, Newsholme P, Soares MJ. The impact of vitamin D levels on inflammatory status: A systematic review of immune cell studies. PLoS One 2015; 10:e0141770.

31. Wu Z, Malihi Z, Stewart AW, Lawes CM, Scragg R. Effect of vitamin D supplementation on pain: A systematic review and meta-analysis. Pain Physician 2016; 19:415-427.

32. Straube S, Derry S, Moore RA, McQuay HJ. Vitamin D for the treatment of chronic painful conditions in adults. Cochrane Database Syst Rev 2010; (1):CDoo7771.

33. Moher D, Liberati A, Tetzlaff J, Altman DG; PRISMA Group. Preferred Reporting Items for Systematic Reviews and Meta-Analyses: The PRISMA statement. Ann Intern Med 2009; 151:264-269, W64.

34. Maher CG, Sherrington C, Herbert RD, Moseley AM, Elkins M. Reliability of the PEDro scale for rating quality of randomized controlled trials. Phys Ther 2003; 83:713-721.

35. Brozek JL, Akl EA, Alonso-Coello P, Lang D, Jaeschke R, Williams JW, Phillips B, Lelgemann M, Lethaby A, Bousquet J, Guyatt GH, Schünemann HJ; GRADE Working Group. Grading quality of evidence and strength of recommendations in clinical practice guidelines. Part 1 of 3. An overview of the GRADE approach and grading quality of evidence about interventions. Allergy 2009; 64:669-677.

36. Abdel Shaheed C, Maher CG, Williams KA, Day R, McLachlan AJ. Efficacy, tolerability, and dose-dependent effects of opioid analgesics for low back pain: A systematic review and meta-analysis. JAMA Intern Med 2016; 176:958-968.

37. Oliveira CB, Franco MR, Maher CG Christine Lin CW, Morelhão PK, Araújo AC, Negrão Filho RF, Pinto RZ. Physical activity tnterventions for increasing objectively measured physical activity levels in patients with chronic musculoskeletal pain: A systematic review. Arthritis Care Res (Hoboken) 2016; 68:1832-1842.

38. Egger M, Davey Smith G, Schneider M, Minder C. Bias in meta-analysis detected by a simple, graphical test. BMJ 1997; 315:629-634.

39. Higgins JPT, Green S (editors). Cochrane Handbook for Systematic Reviews of Interventions Version 5.1.0 [updated March 2011]. The Cochrane Collaboration, 2011. Available from www. handbook.cochrane.org.

40. Balshem $H$, Helfand $M$, Schünemann HJ, Oxman AD, Kunz R, Brozek J, Vist GE, Falck-Ytter Y, Meerpohl J, Norris S, Guyatt GH. GRADE guidelines: 3. Rating the quality of evidence. J Clin Epidemiol 2011; 64:401-406.

41. Ostelo RW, Deyo RA, Stratford P, Waddell G, Croft P, Von Korff M, Bouter $L M$, de Vet HC. Interpreting change scores for pain and functional status in low back pain: Towards international consensus regarding minimal important change. Spine (Phila Pa 1976) 2008; 33:90-94.

42. Higgins JP, Thompson SG, Deeks JJ, Altman DG. Measuring inconsistency in meta-analyses. BM] 2003; 327:557-560.

43. Santos FC, Moraes NS, Pastore A, Cendoroglo MS. Chronic pain in long-lived elderly: Prevalence, characteristics, measurements and correlation with serum vitamin D level. Revista Dor 2015; 16:171-175.

44. Lyritis GP, Androulakis C, Magiasis B, Charalambaki Z, Tsakalakos N. Effect of nandrolone decanoate and 1-alphahydroxy-calciferol on patients with vertebral osteoporotic collapse. A doubleblind clinical trial. Bone Miner 1994; 27:209-217.

45. Iwamoto J, Takeda T, Ichimura S, Matsu K, Uzawa M. Effects of cyclical etidronate with alfacalcidol on lumbar bone mineral density, bone resorption, and back pain in postmenopausal women with osteoporosis. J Orthop Sci 2003; 8:532-537.

46. Knutsen KV, Madar AA, Brekke $M$, Meyer HE, Natvig B, Mdala I, Lagerløv P. Effect of vitamin D on musculoskeletal pain and headache: A randomized, double-blind, placebo-controlled trial among adult ethnic minorities in Norway. Pain 2014; 155:2591-2598.

47. Wandless I, Jarvis S, Evans JG, Aird EG, Stevens J. Vitamin $\mathrm{D}_{3}$ in osteoporosis. $\mathrm{Br}$ Med J 1980; 280:1320.
48. Ota N, Ito H. Comparison of risedronate, raloxifene, calcitonin and active vitamin D treatments in postmenopausal osteoporosis with low back pain. Calcif Tissue Int 2007; 80:S140-S (article P387).

49. Schreuder F, Bernsen RM, van der Wouden JC. Vitamin D st-upplementation for nonspecific musculoskeletal pain in non-western immigrants: A randomized controlled trial. Ann Fam Med 2012; 10:547-555.

50. Majima T, Shimatsu A, Komatsu Y, Satoh N, Fukao A, Ninomiya K, Matsumura T, Nakao K. Effects of risedronate or alfacalcidol on bone mineral density, bone turnover, back pain, and fractures in Japanese men with primary osteoporosis: Results of a two-year strict observational study. J Bone Miner Metab 2009; 27:168-174.

51. Campos TF, Beckenkamp PR, Moseley AM. Usage evaluation of a resource to support evidence-based physiotherapy: The Physiotherapy Evidence Database (PEDro). Physiotherapy 2013; 99:252-257.

52. Garcion E, Wion-Barbot N, MonteroMenei CN, Berger F, Wion D. New clues about vitamin $D$ functions in the nervous system. Trends Endocrinol Metab 2002; 13:100-105.

53. Stumpf WE, O'Brien LP. 1,25 $(\mathrm{OH}) 2$ vitamin $D_{3}$ sites of action in the brain. An autoradiographic study. Histochemistry 1987; 87:393-406.

54. Autier P, Boniol M, Pizot C, Mullie P. Vitamin D status and ill health: A systematic review. Lancet Diabetes Endocrinol 2:76-89.

55. Grossmann RE, Zughaier SM, Liu S, Lyles RH, Tangpricha V. Impact of vitamin D supplementation on markers of inflammation in adults with cystic fibrosis hospitalized for a pulmonary exacerbation. Eur J Clin Nutr 2012; 66:1072-1074.

56. Hopkins MH, Owen J, Ahearn T, Fedirko V, Flanders WD, Jones DP, Bostick RM. Effects of supplemental vitamin D and calcium on biomarkers of inflammation in colorectal adenoma patients: A randomized, controlled clinical trial. Cancer Prev Res (Phila) 2011; 4:1645-1654.

57. Rosendahl-Riise H, Spielau U, Ranhoff AH, Gudbrandsen OA, Dierkes J. Vitamin D supplementation and its influence on muscle strength and mobility in community-dwelling older persons: A systematic review and meta-analysis. J Hum Nutr Diet 2017; 30:3-15. 
58. Stockton KA, Mengersen K, Paratz JD, Kandiah D, Bennell KL. Effect of vitamin D supplementation on muscle strength: A systematic review and meta-analysis. Osteoporos Int 2011; 22:859-871.

59. Ghai B, Bansal D, Kanukula R, Gudala K, Sachdeva N, Dhatt SS, Kumar V. Vitamin $D$ supplementation in patients with chronic low back pain: An open label, single arm clinical trial. Pain Physician 2017; 20:E99-E105.

6o. Fujita T, Fujii Y, Munezane H, Ohue M, Takagi Y. Analgesic effect of raloxifene on back and knee pain in postmenopausal women with osteoporosis and/or osteoarthritis. J Bone Miner Metab 2010; 28:477-484.

61. Bjelakovic G, Gluud LL, Nikolova D, Whitfield K, Wetterslev J, Simonetti RG, Bjelakovic M, Gluud C. Vitamin D supplementation for prevention of mortality in adults. Cochrane Database Syst Rev 2014; (1):CDoo7470.

62. Ringe JD, Farahmand P, Schacht E, Rozehnal A. Superiority of a combined treatment of alendronate and alfacalcidol compared to the combination of alendronate and plain vitamin D or alfacalcidol alone in established postmenopausal or male osteoporosis (AAC-Trial). Rheumatol Int 2007; 27:425-434.

63. Narula N, Cooray M, Anglin R, Muqta$\operatorname{dir}$ Z, Narula A, Marshall JK. Impact of high-dose vitamin $D_{3}$ supplementation in patients with Crohn's disease in remission: A pilot randomized doubleblind controlled study. Dig Dis Sci 2017; 62:448-455

64. Zaleski A, Panza G, Swales H, Arora P, Newton-Cheh C, Wang T, Thompson PD, Taylor B. High-dose versus lowdose vitamin $D$ supplementation and arterial stiffness among individuals with prehypertension and vitamin $D$ deficiency. Dis Markers 2015; 2015:918968.

65. Burdorf A, Sorock G. Positive and negative evidence of risk factors for back disorders. Scand J Work Environ Health 1997; 23:243-256.
66. Holick MF, Binkley NC, Bischoff-Ferrari HA, Gordon CM, Hanley DA, Heaney RP, Murad MH, Weaver CM; Endocrine Society. Evaluation, treatment, and prevention of vitamin $D$ deficiency: An Endocrine Society clinical practice guideline. J Clin Endocrinol Metab 2011; 96:1911-1930.

67. Gröber U, Spitz J, Reichrath J, Kisters K, Holick MF. Vitamin D: Update 2013: From rickets prophylaxis to general preventive healthcare. Dermatoendocrinol 2013; 5:331-347.

68. Tanaka R, Ozawa J, Kito N, Moriyama H. Efficacy of strengthening or aerobic exercise on pain relief in people with knee osteoarthritis: A systematic review and meta-analysis of randomized controlled trials. Clin Rehabil 2013; 27:1059-1071.

69. Kamper SJ, Maher CG, Hancock MJ, Koes BW, Croft PR, Hay E. Treatmentbased subgroups of low back pain: A guide to appraisal of research studies and a summary of current evidence. Best Pract Res Clin Rheumatol 2010; 24:181-191.

70. Saragiotto BT, Maher CG, Moseley AM, Yamato TP, Koes BW, Sun X, Hancock MJ. A systematic review reveals that the credibility of subgroup claims in low back pain trials was low. J Clin Epidemiol 2016; 79:3-9..

71. Lotfi A, Abdel-Nasser AM, Hamdy A, Omran AA, El-Rehany MA. Hypovitaminosis $D$ in female patients with chronic low back pain. Clin Rheumatol 2007; 26:1895-1901.

72. Abdulmonem A, Hanan A, Elaf A, Haneen $T$, Jenan $A$. The prevalence of musculoskeletal pain \& its associated factors among female saudi school teachers. PakJ Med Sci 2014; 30:1191-1196.

73. Madani M, Alavi NM, Taghizadeh M. Non-specific musculoskeletal pain and vitamin $D$ deficiency in female nurses in Kashan, Iran. J Musculoskelet Pain 2014; 22:268-274.

74. Zadro J, Shirley D, Ferreira M, Carvalho-
Silva AP, Lamb SE, Cooper C, Ferreira $\mathrm{PH}$. Mapping the association between vitamin $d$ and low back pain: A systematic review and meta-analysis of observational studies. Pain Physician 2017; 20:611-640.

75. e Silva AV, Lacativa PG, Russo LA, de Gregório LH, Pinheiro RA, Marinheiro LP. Association of back pain with hypovitaminosis $D$ in postmenopausal women with low bone mass. BMC Musculoskelet Disord 2013; 14:184.

76. Hicks GE, Shardell M, Miller RR, Bandinelli S, Guralnik J, Cherubini A, Lauretani F, Ferrucci L. Associations between vitamin $D$ status and pain in older adults: The Invecchiare in Chianti study. J Am Geriatr Soc 2008; 56:785-791.

77. Tanaka S, Kuroda T, Yamazaki Y, Shiraki Y, Yoshimura N, Shiraki M. Serum 25-hydroxyvitamin $D$ below $25 \mathrm{ng} / \mathrm{mL}$ is a risk factor for long bone fracture comparable to bone mineral density in Japanese postmenopausal women. J Bone Miner Metab 2013; 32:514-523.

78. Lodh M, Goswami B, Mahajan RD, Sen D, Jajodia N, Roy A. Assessment of vitamin $D$ status in patients of chronic low back pain of unknown etiology. Indian J Clin Biochem 2015; 30:174-179.

79. Prakash S, Kumar M, Belani P, Susvirkar A, Ahuja S. Interrelationships between chronic tension-type headache, musculoskeletal pain, and vitamin $\mathrm{D}$ deficiency: Is osteomalacia responsible for both headache and musculoskeletal pain? Ann Indian Acad Neurol 2013; 16:650-658.

8o. Chatterjee R, Hemmings S, Laupheimer MW. The effect of low vitamin D on chronic non-specific low back pain: A systematic review. Int Musculoskelet Med 2016; 2016:1-8.

81. Hopewell S, Clarke M, Mallett S. Grey literature and systematic reviews. In: Rothstein HR, Sutton AJ, Borenstein M (eds). Publication Bias in Meta-Analysis. John Wiley \& Sons, Ltd, Hoboken 2006, pp 49-72. 
